OPEN ACCESS

Edited by:

Gustavo Henrique Goldman,

University of São Paulo, Brazil

Reviewed by:

Yulong Wang,

Anhui Agricultural University, China

Xingzhong Liu,

Institute of Microbiology (CAS), China

*Correspondence:

Li-Qiong Guo

guolq@scau.edu.cn

Jun-Fang Lin

linjf@scau.edu.cn

${ }^{\dagger}$ These authors have contributed equally to this work

Specialty section:

This article was submitted to Fungi and Their Interactions,

a section of the journal

Frontiers in Microbiology

Received: 02 January 2020

Accepted: 16 March 2020

Published: 24 April 2020

Citation:

Chen B-X, Wei T, Xue L-N, Zheng $Q-W$, Ye $Z-W$, Zou Y, Yang $Y$, Yun F, Guo L-Q and Lin J-F (2020)

Transcriptome Analysis Reveals the Flexibility of Cordycepin Network in Cordyceps militaris Activated by

L-Alanine Addition.

Front. Microbiol. 11:577.

doi: 10.3389/fmicb.2020.00577

\section{Transcriptome Analysis Reveals the Flexibility of Cordycepin Network in Cordyceps militaris Activated by L-Alanine Addition}

\author{
Bai-Xiong Chen 1,2t, Tao Wei 1,2t, Ling-Na Xue 1,2, Qian-Wang Zheng ${ }^{1,2}$, Zhi-Wei Ye ${ }^{1,2}$, \\ Yuan Zou ${ }^{1,2}$, Yi Yang ${ }^{1,2}$, Fan Yun ${ }^{3}$, Li-Qiong Guo ${ }^{1,2 *}$ and Jun-Fang Lin ${ }^{1,2 *}$ \\ 1 Institute of Food Biotechnology \& College of Food Science, South China Agricultural University, Guangzhou, China \\ ${ }^{2}$ Research Center for Micro-Ecological Agent Engineering and Technology of Guangdong Province, Guangzhou, China, \\ ${ }^{3}$ Guangzhou Alchemy Biotechnology Co., Ltd., Guangzhou, China
}

Cordycepin, isolated from the traditional medicinal fungus Cordyceps militaris, has gained much attention due to its various clinical functions. Previous reports of L-alanine addition could significantly improve cordycepin production, but the molecular mechanism remains unknown. In this study, transcriptome analysis of $C$. militaris with doubled cordycepin production induced by L-alanine addition provides an insight into the flexibility of the cordycepin network. The biopathways of energy generation and amino acid conversion were activated so that cordycepin substrate generation was consequently improved. Specific genes of rate-limiting enzymes in these pathways, as well as related transcription factors, were figured out. Two key Zn2Cys6-type transcription factors $\mathrm{CmTf1}$ and $\mathrm{CmTf2}$ were verified to play the roles of doubling the cordycepin production by overexpression of their coding genes in $C$. militaris wild type. These results provide a complete map of the cordycepin network in $C$. militaris with a distinct understanding of the flexibility of joints, giving a better foundation for increasing cordycepin yield and strain breeding in the future.

Keywords: cordycepin, Cordyceps militaris, metabolic network, flexibility, transcription factor

\section{INTRODUCTION}

Cordyceps militaris has been popular in East Asia for centuries as a kind of edible and medicinal fungus. Recently, the application of various bioactive metabolites isolated from C. militaris led to rapid development in food, pharmaceutical, and cosmetics industries (Paterson, 2008; Lee et al., 2009; Zhou et al., 2009). To extend the applications of C. militaris, its bioactive metabolites, such as adenosine, ergosterol, ergothioneine (Cohen et al., 2014), Cordyceps polysaccharides (Zhang et al., 2015), and cordycepin (Cunningham et al., 1950; Paterson, 2008), are extracted and made into tablets and capsules. Among these biologically active ingredients, cordycepin has attracted the most attention for its multiple therapeutic effects (Tuli et al., 2014). Cordycepin ( $3^{\prime}$-deoxyadenosine) was proved to be able to inhibit cell proliferation and induce cell apoptosis (Tian et al., 2015) by

Abbreviations: HPLC, high-performance liquid chromatography; DEGs, differentially expressed genes; FDR, false discovery rate; FPKM, fragments per kilo-base of exon model per million mapped reads; GO, Gene Ontology; KEGG, Kyoto Encyclopedia of Genes and Genomes; TF, transcription factor; ABC, ATP-binding cassette. 
binding signaling molecules, which led to anti-inflammatory effect (Noh et al., 2008). By preventing excessive glutamateinduced oxidation and endoplasmic reticulum stress-associated apoptosis in hippocampal cells (Jin et al., 2014), it was also proved to have antioxidant effects (Kim et al., 2006). Besides, cordycepin was proved to have broad-spectrum antibiotic activity by inhibiting $\mathrm{NAD}^{+}$-dependent DNA ligase (Zhou et al., 2016).

To optimize the production of this medical component, various kinds of energy sources (Mao et al., 2005; Mao and Zhong, 2006), putative precursors (Gu et al., 2007), mineral salts (Le et al., 2006; Fan et al., 2012), and culture conditions (Masuda et al., 2007; Kang et al., 2014) in C. militaris culturing had been tested. The highest production of cordycepin was reported by adding $\mathrm{L}$-alanine in liquid static $C$. militaris fermentation, yielding up to $2 \mathrm{~g} / \mathrm{L}$ (Kang et al., 2014). However, a detailed mechanism at the genetic level is still rarely reported.

High-throughput sequencing technologies have been used to discover the biosynthetic and metabolic pathway of cordycepin (Zheng et al., 2011; Yin et al., 2012; Raethong et al., 2018). As an isomer of $2^{\prime}$-deoxyadenosine, cordycepin was predicted to share a similar synthesis pathway of $2^{\prime}$-deoxyadenosine, but the relevant key enzyme was missing in the genome of $C$. militaris (Zheng et al., 2011). This revealed that cordycepin might have a different metabolic pathway. The cordycepin biosynthetic pathway was narrowed in the purine metabolism pathway by transcriptome and proteome analysis of two different stages of $C$. militaris (Yin et al., 2012). A three-gene biosynthesis cluster was reported (Xia et al., 2017) to synthesize cordycepin from adenosine, and it revealed that cordycepin metabolism overlapped with parts of the purine pathway. Further transcriptome analysis of samples with three carbon sources (Raethong et al., 2018) had tried to draw a network between adenosine, methionine, and cordycepin. Unfortunately, it failed to provide further verification in a molecular level.

To better understand the molecular mechanism of L-alanine's action on cordycepin production, a transcriptome analysis was carried out in this study. The transcription data revealed key ratelimiting enzymes and transcription factors with high flexibility in cordycepin synthesis, and a metabolic network map from the substrate amino acid to the product cordycepin in C. militaris was drawn. In this study, two key Zn2Cys6-type transcription factors CmTf1 and CmTf2, which were highly transcribed after the adding of L-alanine, were overexpressed and verified to be the function of improving the cordycepin yield in vivo. Improved production of cordycepin in CmTf1/2 overexpressed strains reveal that the $\mathrm{Zn} 2 \mathrm{Cys} 6$-type transcription factors (TFs) were not only involved in the development of $C$. militaris fruiting (Zheng et al., 2011) but also joined in the regulation of secondary metabolites in C. militaris.

\section{MATERIALS AND METHODS}

\section{Culture Conditions}

Two types of media were modified from a previous report (Kang et al., 2014). A basal medium, containing (g/L) peptone 20, sucrose 24.7, $\mathrm{K}_{2} \mathrm{HPO}_{4} \cdot 3 \mathrm{H}_{2} \mathrm{O} 1.11, \mathrm{MgSO}_{4} \cdot 7 \mathrm{H}_{2} \mathrm{O} 0.90$, and
Vitamin $\mathrm{B}_{1}$ 0.01, was used and $8 \mathrm{~g} / \mathrm{L}$ L-alanine was added as treatment. C. militaris was incubated on Potato Dextrose Agar slants at $25^{\circ} \mathrm{C}$ for 7 days to form mycelium pellets. The pellets were transferred into a $250 \mathrm{ml}$ flask contained $70 \mathrm{ml}$ basal medium and cultured at $150 \mathrm{rpm} \mathrm{min} \mathrm{min}^{-1}$ for 8 days to expand the culture. The liquid static fermentation was performed in a 1$\mathrm{L}$ wide-mouth glass jar (Figure 1A), $30 \mathrm{ml}$ mature C. militaris CM10 mycelium pellets mixed with $270 \mathrm{ml}$ of basal medium or its treatment, to build the samples of CMsI or CMsII. The jars maintained stationary in the dark at $25^{\circ} \mathrm{C}$ for 30 days. Mycelium layer on the surface from the sample was collected, frozen, and dried.

Since the static fermentation of $C$. militaris needs to take a longer time and harder to make samples equal, the shakeflask fermentation was performed to verify the impact of in vivo TF overexpression on cordycepin production. The mycelium of C. militaris CM10Tf1 and CM10Tf2 was collected from fresh plates and inoculated into $100 \mathrm{ml}$ of PPDB (potato extracts $200 \mathrm{~g} / \mathrm{L}$, glucose $20 \mathrm{~g} / \mathrm{L}, \mathrm{MgSO}_{4} \cdot 7 \mathrm{H}_{2} \mathrm{O} 1.5 \mathrm{~g} / \mathrm{L}, \mathrm{KH}_{2} \mathrm{PO}_{4} 3 \mathrm{~g} / \mathrm{L}$, peptone $10 \mathrm{~g} / \mathrm{L}, \mathrm{VB} 110 \mathrm{mg} / \mathrm{L})$ medium. After fermentation at $25^{\circ} \mathrm{C}$ with $150 \mathrm{rpm}$ for 5 days, the medium was supplemented with $8 \mathrm{~g} / \mathrm{L}$ L-alanine and continued to shake for 3 days before the harvest.

\section{HPLC Detection of Cordycepin}

After fermentation, the mycelia were lyophilized and ground to powder. Two grams of dry powder was weighted from each sample to perform HPLC detection. HPLC assay was performed on Model LC2000 Liquid Chromatography System (TECHCOMP, Shanghai, China) equipped with an ULTIMATE AQ-C18 HPLC Column $(4.6 \times 250$ mm, 5-Micron; Welch, Shanghai, China). The analysis conditions were as follows: mobile phase, $85 \%$ ultra-pure water and 15\% methanol (v/v); flow rate, $1 \mathrm{ml} / \mathrm{min}$; detection wavelength, $260 \mathrm{~nm}$; injection volume, 20 $\mu 1$. A standard cordycepin curve was generated using $0.02-0.25$ $\mu \mathrm{g} / \mathrm{ml}$ cordycepin standard (Sigma-Aldrich, United States). The cordycepin yield was calculated using the detected peak area according to the standard curve. The cordycepin concentration of mycelia presented in the study was calculated by normalizing in the equal biomass.

\section{Extraction of Total RNA, Library Establishment, and Transcriptome Sequencing}

Total RNA was extracted from $100 \mathrm{mg}$ of frozen mycelium pellets using the E.Z.N.A. Fungal RNA Miniprep Kit (OMEGA BioTek, United States). After DNase I treatment, the quality of RNA was assessed by a NanoDrop and Agilent 2100. Oligo(dT) was used to isolate mRNA. The mRNAs were fragmented and used as templates to synthesize cDNA. Short fragments were purified and processed by end repair, single-nucleotide adenine addition, and adapter connection. Suitable fragments were selected for PCR amplification. The quantification and qualification of the sample library were performed by an Agilent 2100 Bioanalyzer (Agilent, Beijing, China) and an ABI StepOnePlus Real-Time PCR System (Thermo Fisher Scientific, United States), and further analyzed 
A
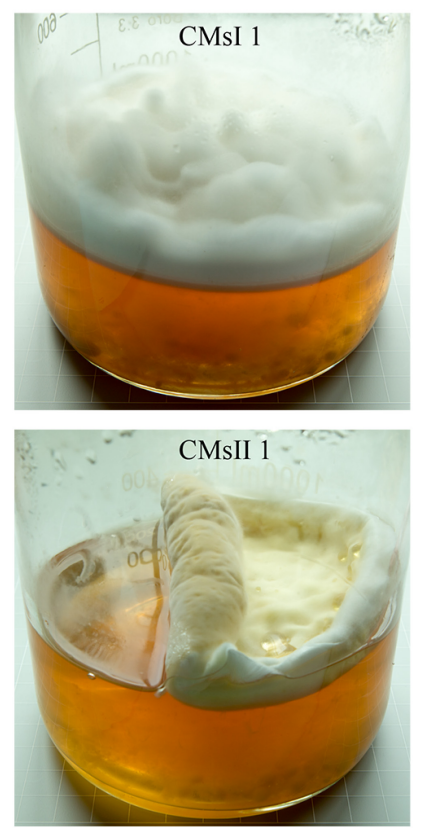

B
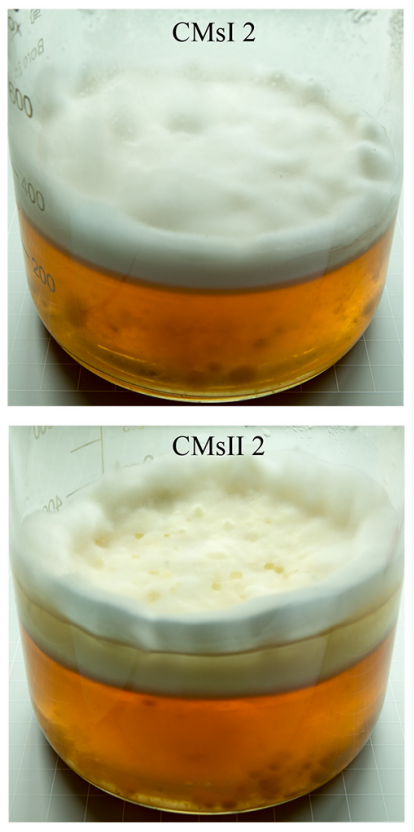

250

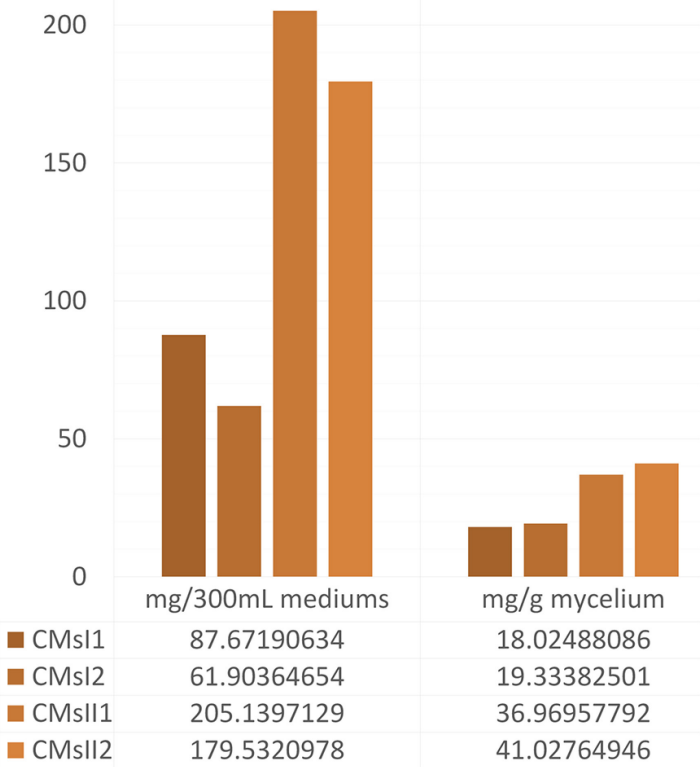

FIGURE 1 | Characteristics of the four studied samples. (A) Growth characteristics of the four samples in glass jars after 30 days of culturing. (B) Cordycepin production in each glass jar.

by following the operating instruction. The library was sequenced using the Illumina HiSeq by BGI (Shenzhen, China).

\section{Mapping the RNA-Seq Reads and Quantitative Analysis of the Genes}

After sequencing, to obtain clean reads, raw reads were filtered for low-quality (the percentage of base whose quality is lesser than 15 is greater than $20 \%$ in a read), adaptor-polluted reads, and reads with a high content of unknown bases (unknown bases are more than 5\%) using BGI (Shenzhen, China) in-house software with the quality system phred64 were removed and clean reads were stored in FASTQ (Cock et al., 2010) format. Clean reads were uploaded to NCBI (Submission ID: PRJNA413637) and mapped to the reference genome C. militaris CM01 (NCBI accession number. AEVU00000000) using HISAT (version: v0.1.6-beta; parameters: -phred64 -sensitive -no-discordant -no-mixed -I 1 -X 1000) (Kim et al., 2015). StringTie (Pertea et al., 2015) (version: v1.0.4; parameters: -f0.3 -j3 -c 5 -g100 -s 10000 -p8) and CuffCompare (Trapnell et al., 2012) (version: v2.2.1; parameters: -p12) were used to reconstruct the transcripts and compare with the reference annotation, respectively. CPC (Kong et al., 2007) (version: v0.9-r2; parameters: default) software was further used to predict the potential novel coding transcripts. These predicted transcripts were merged with the reference transcripts to obtain a complete reference. For gene expression analysis, clean reads were mapped to the complete reference using Bowtie2 (Langmead and Salzberg, 2012) (version: v2.2.5; parameters: -q phred64 -sensitive -dpad0 -gbar99999999 -mp1, 1 -np1 -score
-minL, 0, -0.1 -I1 -X 1000 -no-mixed -no-discordant -p1 -k 200) and analyzed with RSEM (Li and Dewey, 2011) (version: v1.2.12; parameters: default), which could be used to estimate gene and isoform expression levels from RNA-Seq data, to obtain the gene expression level for each sample. The gene expression level was determined by calculating the number of fragments in each sample and normalized to FPKM (Fragments Per Kilo-base of exon model per Million mapped reads).

\section{GO Functional Classification and KEGG Pathway Enrichment Analysis of DEGs}

Differentially expressed genes (DEGs) were detected using edge R criteria in R (Robinson et al., 2010) according to the RSEM results. The detection of DEGs between two treatments (CMsI vs. CMsII) was based on the noisy distribution method (NOIseq) (Tarazona et al., 2011), and the corrected $P>0.8$ and absolute value of fold change $>2$ were considered as DEGs, while detection of DEGs between every single sample (CMsI1 vs. CMsI2 vs. CMsII1 vs. CMsII2) was based on the Poisson distribution method (PoissonDis) (Audic and Claverie, 1997), and the false discovery rate, FDR $<0.001$, and the absolute value of fold change $>2$ were considered as DEGs. To further analyze the DEG annotations, Gene Ontology (GO) functional classification and a Kyoto Encyclopedia of Genes and Genomes (KEGG) pathway enrichment analysis were carried out based on the GO database (Ashburner et al., 2000) and KEGG database (Kanehisa et al., 2007). 


\section{Quantitative Real-Time PCR Validations of DEGs}

The qRT-PCR template cDNA was synthesized from $0.5 \mu \mathrm{g}$ of total RNA by ReverTra Ace qPCR RT Master Mix (Toyobo, Osaka, Japan). All primers used for the quantitative realtime PCR (qRT-PCR) are listed in Supplementary Table S1. Each qRT-PCR reaction system had a total volume of $20 \mu \mathrm{l}$, containing $50 \mathrm{ng}$ of cDNA, $160 \mathrm{nM}$ relevant primers, and the SYBR Green Realtime PCR Master Mix (Toyobo). All qRTPCR reactions were carried out in the CFX96 Real-Time PCR Detection System (Bio-Rad, CA, United States), following the reaction parameters in the instruction book from the SYBR Green Realtime PCR Master Mix (Toyobo, Osaka, Japan). Using the elongation factor 1-alpha (tef1) gene (NW_006271969.1) as an internal control for each sample (Lian et al., 2014), the relative gene expression levels were calculated by the $2^{-\Delta \Delta}$ CT method (Livak and Schmittgen, 2001).

\section{Overexpression of Putative Transcription Factors}

The transcription factors CCM_02568 gene (accession number NW_006271970.1) and CCM_01481 (accession number NW_006271969.1) gene were cloned from C. militaris CM10 by primers Tf1F/Tf1R and Tf2F/Tf2R (Supplementary Table S10). These genes were renamed as $c m t f 1 / \mathrm{cm} t f 2$ (represented the full-length gene of CCM_02568/CCM_01481, respectively) and constructed into plasmid p390-blpR-sgRNA-cmcas9-gfp (Chen et al., 2018) by XbaI/BcuI using a ClonExpress II One Step Cloning Kit (Vazyme Biotech Co., Ltd, Nanjing, China) to build p390-blpR-CmTf1/2. Then the overexpressed vectors were transformed into Agrobacterium tumefaciens AGL-1 (Weidi Bio, Shanghai, China) by electroporation. The A. tumefaciensmediated transformation (ATMT) was used to build the CmTf1/2 overexpression $C$. militaris as described in a previous study (Chen et al., 2018).

\section{RESULTS}

\section{Growth Characteristics and Cordycepin Production From C. militaris After L-Alanine Addition}

After 30 days of liquid culturing, mycelia and the color of residual medium did not present any significant or sudden change, indicating that large-scale cell autolysis did not occur in the fermentation, and the amount of carbon source and nitrogen source in the medium was enough for the long period of mycelia growth. In static liquid culture, compared to the control, primordium in the CMsII group was visually thicker (Figure 1A). According to the standard curve formula, $y=1 \mathrm{e}-08 x+0.001\left(R^{2}=0.9994\right)$, the cordycepin extraction yield detected by HPLC was obtained. The total amount of 36.9 and $41.0 \mathrm{mg} / \mathrm{g}$ of cordycepin was extracted from the dry mycelia harvested from the CMsI group, while the other group was 18.0 and $19.3 \mathrm{mg} / \mathrm{g}$ (Figure 1B). After adding L-alanine in the static liquid culture, the cordycepin concentration increased 2.1 times.

\section{Sequencing Data Analysis and Annotation}

Four cDNA samples from C. militaris mycelia were processed by the Illumina HiSeq platform and, on average, generated 4.44 $\mathrm{Gb}$ of clean bases in each sample (Supplementary Table S2). After mapping the sequenced reads to the reference genome, on average $80.3 \%$ of the reads were mapped (Supplementary Table S3). After reconstruction, 4573 novel transcripts were predicted in all four samples. Among these, 4013 transcripts were previously unknown splicing events for known genes, while 68 were novel coding transcripts without any known features, and the remaining 492 were long non-coding RNAs.

After the novel coding transcripts were merged with the reference transcript to obtain a complete reference, clean reads were mapped to this reference (Supplementary Table S4) to calculate the gene expression level for each sample. Based on the mapping results, the read distributions, the Pearson correlation coefficient between expression values across samples and hierarchical clustering between four samples were calculated (Figures 2A-C and Supplementary Figure S1).

\section{DEGs in C. militaris}

Global transcriptional changes from normalizing the DEG data were analyzed to identify DEGs (Figure 2D). Compared to the treatment without the addition of L-alanine, there were 511 genes up-regulated and 472 genes down-regulated in the treatment (CMsI vs. CMsII) (Figure 2E). A volcano plot was drawn to show the global fold changes between the upregulated and down-regulated genes (Figure 2F). As shown in the volcano plot, most of the DEGs represented a slight fold change, while some of the DEGs displayed significant differences. Results with this pattern revealed that adding L-alanine may regulate some unknown genes in the cordycepin metabolic network. Additionally, a heatmap was drawn to present the hierarchical clustering of the DEGs among samples (Figure 2G). As shown in the heatmap, the expression profiles between the replicates were similar, indicating that the transcriptome data were reliable.

\section{GO Analysis and KEGG Pathway Enrichment of DEGs}

GO classification and functional enrichment were performed for the gene annotation. A total of 523, 338, and 279 DEGs were assigned to the ontologies "biological process," "molecular function," and "cellular component," respectively (Figure 3A). Over 100 DEGs were classified into five GO terms, including "cellular process," "metabolic process," "singleorganism process," "binding," and "catalytic activity." It indicated that a certain amount of DEGs was predicted to be correlated with transmembrane transport and signal transduction in the GO database. To further verify the GO ontologies results, a GO functional enrichment of the DEGs was performed and displayed by software Cytoscape (Shannon et al., 2003) with 


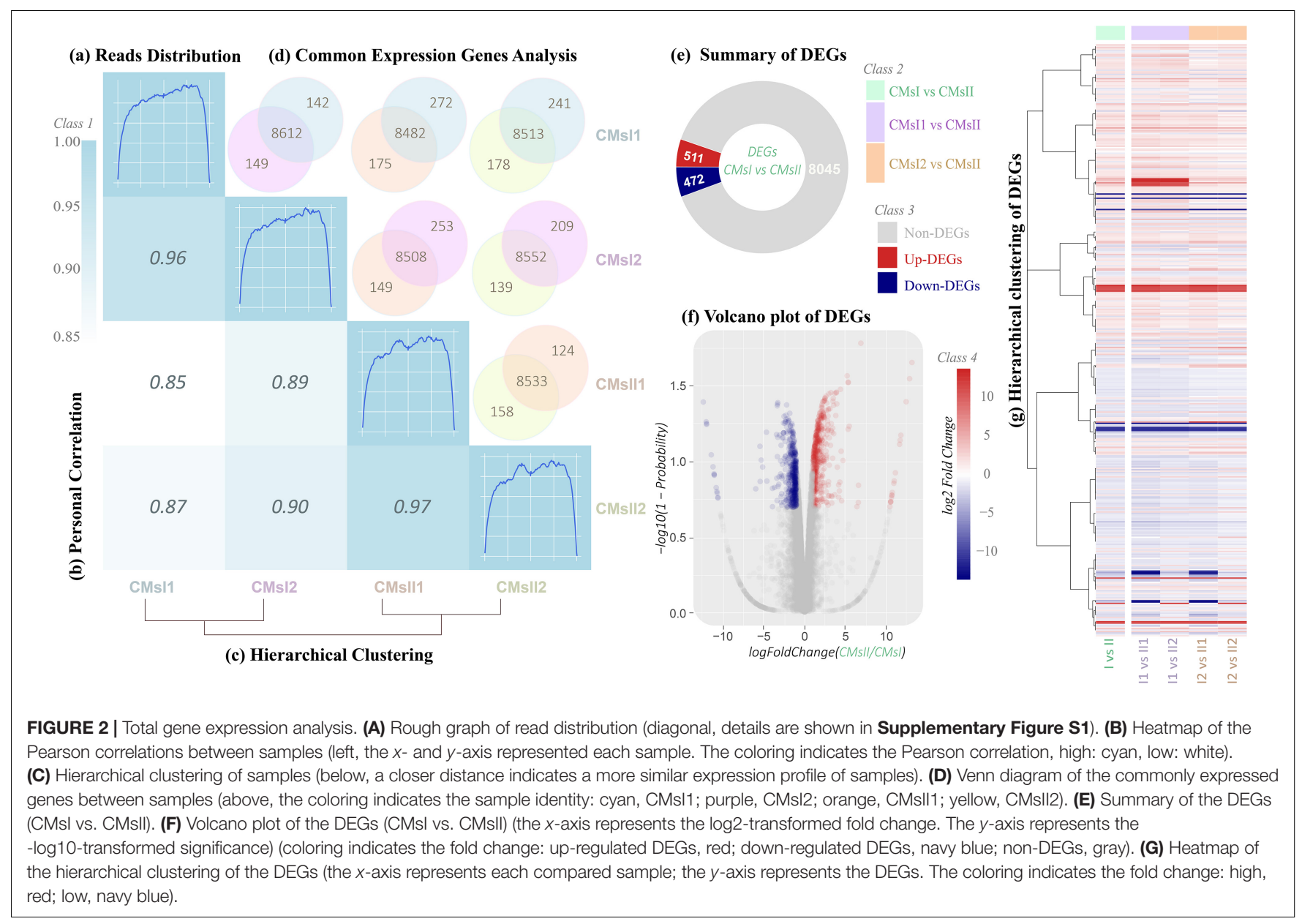

package BiNGO (Maere et al., 2005; Figure 3B). Terms in Figure 3B showing the smallest corrected $P$-value indicated that the Biological Process of transmembrane transport was significantly enriched in the DEGs $(P<0.01)$, suggesting its importance in the metabolism of supplementing L-alanine.

To have a better understanding of the interactions of functional genes by pathway-based analysis, all the genes were mapped to the KEGG database to categorize the biological functions of the DEGs. Specific gene enrichment was observed in 118 pathways. Twenty-one pathways were showing DEG enrichment (965 genes), and the cluster "Global and overview maps" was the largest group (241 genes, 25.0\%). Clusters for "Amino acid metabolism" (98 genes, 10.2\%), "Carbohydrate metabolism" (96 genes, 9.9\%), “Transport and catabolism" (70 genes, 7.3\%), "Signal transduction" (57 genes, $6.0 \%)$, and "Translation" (57 genes, 6.0\%) were followed (Figure 3C). Moreover, the $P$-values of 12 pathways were less than 0.05 (Table 1). The pathways "Glycine, serine and threonine metabolism," "Metabolic pathways," and "Starch and sucrose metabolism" showed an even greater significant enrichment $(P<0.01)$.

DEGs that took part in the pathway of autophagy regulation (KEGG pathway ko04140) were not shown enrichment, indicating that large-scale cell starvation did not occur in the fermentation. It also indicated that the increased yield of cordycepin was caused by the interaction affection of L-alanine in the metabolic network. A network map of substance interactions affected by L-alanine addition was obtained by the comparison between the transcriptome data and the KEGG database (Figure 4 and Supplementary Table S5). Interactions between substances were directed by the DEGs and eventually combined to draw one transformation network.

As shown in the network, L-cysteine and L-glutamate played key roles in substance transformation after adding L-alanine. Other amino acids, such as glutamine, glycine, and L-aspartate, which played an important role in this network had been reported to be involved in the purine pathway (Zheng et al., 2011). It indicated that additives like L-alanine may improve cordycepin production by interfering with amino acid conversion. Also, according to the comparison with KEGG pathway ko01100, significant transcriptional changes of genes were enriched in the pathways of the glycolysis of carbohydrate and amino acid metabolism, pentose phosphate pathway and citrate cycle of energy metabolism, the fatty acid pathway, and ribonucleotide metabolism (Supplementary Figure S2 and Supplementary Table S6). 


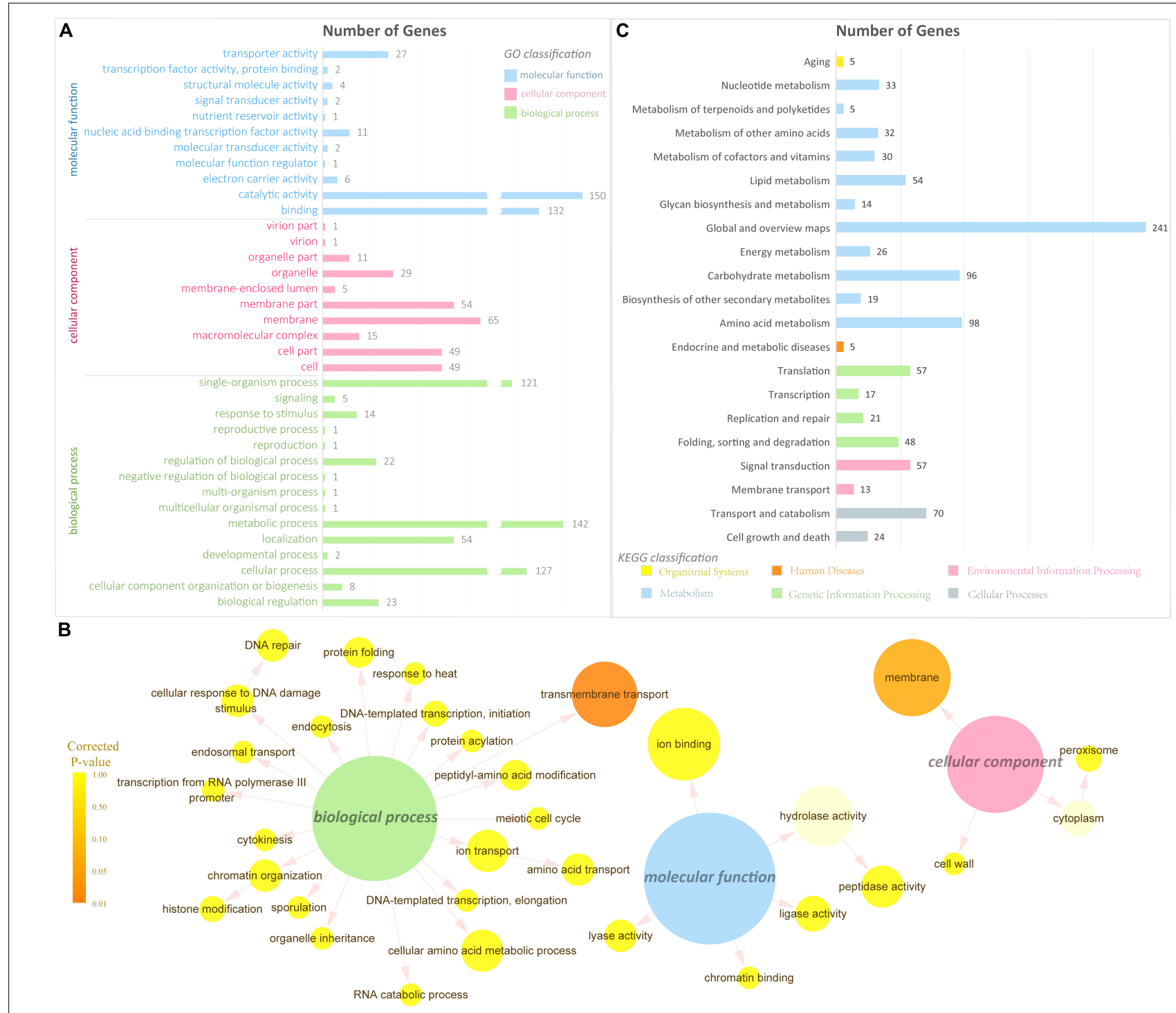

FIGURE 3 | Gene Ontology and pathway analysis. (A) GO classification of the DEGs (the $x$-axis represents the number of DEGs, and the $y$-axis represents the GO term). (B) GO enrichment of the DEGs. The color of the column indicates the corrected P-value: (high, yellow; low, orange) or the ontology (blue, molecular function; pink, cellular component; green, biological process). (C) Pathway classification of the DEGs (the $x$-axis represents the number of DEGs, and the $y$-axis represents the pathway name).

\section{Putative Transcription Factors Involved in Amino Acid Metabolism and Production of Cordycepin}

To further analyze the molecular interaction mechanism between L-alanine addition and cordycepin overproduction, the amino acid sequence of DEGs between CMsI and CMsII were uploaded into the Fungal Transcription Factor Database (Park et al., 2008) (FIFD $)^{1}$ to detect putative transcription factors. Output candidates were further verified by InterPro ${ }^{2}$. A total of 94 putative TFs from 983 DEGs (10.5\%) was predicted and

${ }^{1}$ http://ftfd.snu.ac.kr/intro.php

${ }^{2}$ https://www.ebi.ac.uk/interpro/ classified into different TF families. These TF families contained Zn2Cys6 (MacPherson et al., 2006), HMG (Hall et al., 2006), Homeodomain-like (Kim et al., 2009), OB-fold nucleic acid binding (Taylor et al., 1995), C2H2 Zinc finger (Kim et al., 2009), Helix-turn-helix (Gallegos et al., 1997), bZIP (Dementhon et al., 2004), Winged helix repressor DNA-binding (Gajiwala and Burley, 2000), etc. (Table 2 and Supplementary Table S7).

The largest family was Zn2Cys6, followed by HMG and Homeodomain-like families. The significantly up-regulated TFs were the Zn2Cys6-type genes CCM_02568 (transcription level, CMsI: 1040.0, CMsII: 2750.2, 1.4-fold increased) and CCM_01481 (CMsI: 255.8, CMsII: 583.3, 1.2-fold increased). A previous work reported (Zheng et al., 2011) that the Zn2Cys6 TF genes CCM_01809 and CCM_09644 were relevant to fruit 
TABLE 1 | The smallest $P$-value $(P<0.05)$ pathway in KEGG.

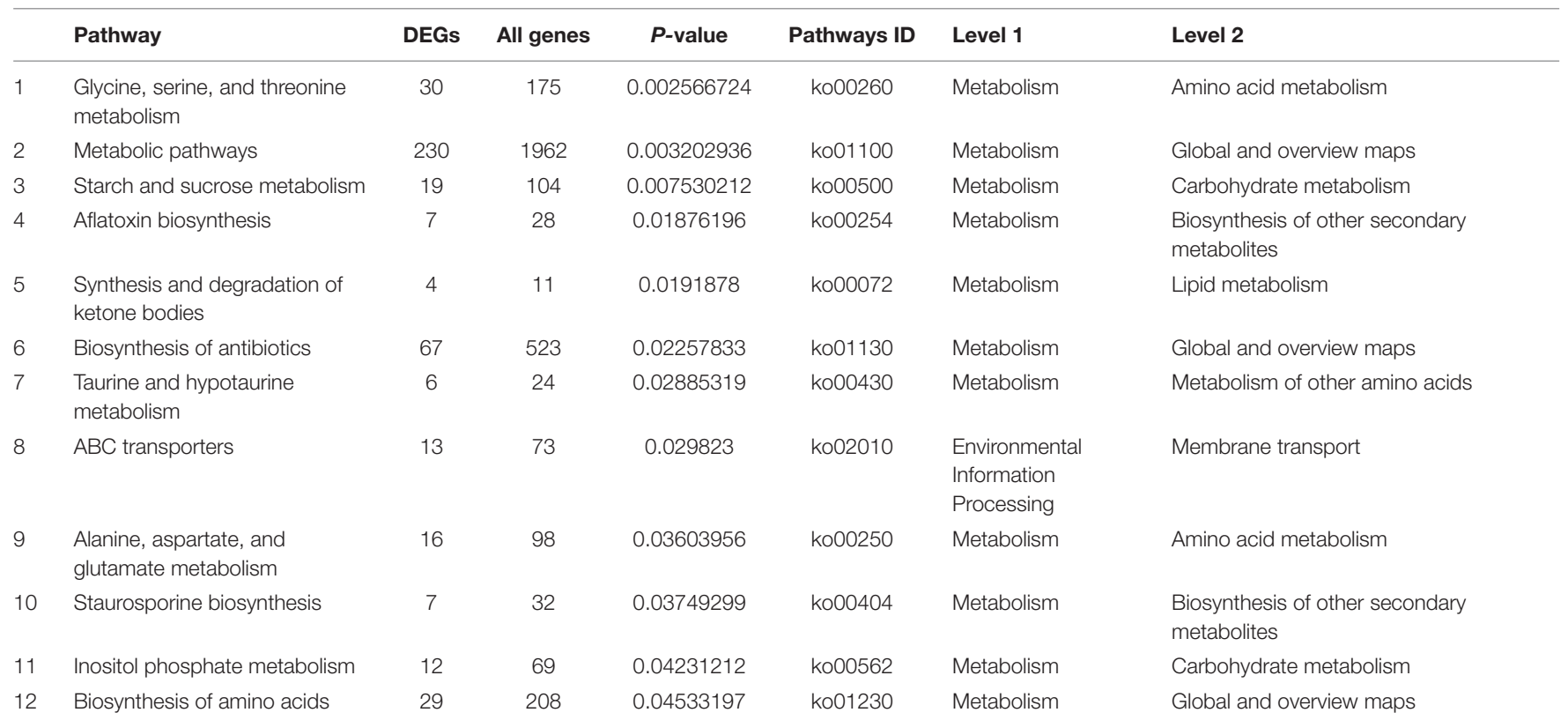

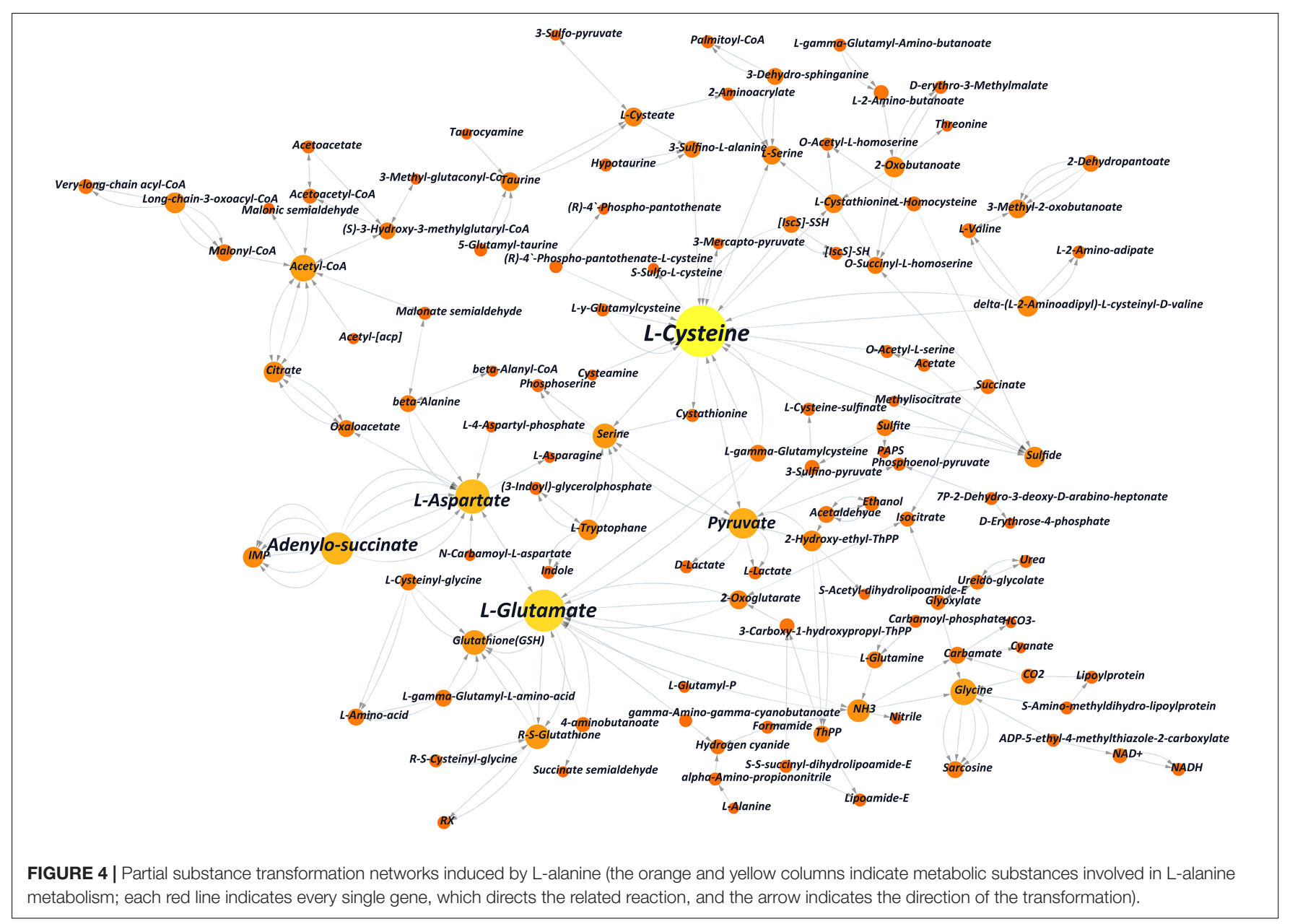


body formation; nevertheless, the Zn2Cys6-type TFs might also play an important role in the amino acid metabolism or cordycepin production.

\section{Putative Cordycepin Metabolism Pathway}

Since the cordycepin production was significantly improved by L-alanine addition, DEGs related to cordycepin synthesis were carefully identified by comparison to the previously reported purine metabolic pathway constructed by Zheng et al. (2011), and Vongsangnak et al. (2017), and the cordycepin biosynthesis gene cassette presented by Xia et al. (2017). Finally, the whole cordycepin metabolic network in C. militaris was predicted (Figure 5). The detailed decipher of hypothetical genes (Table 3) involved in the network was as follows.

To begin with the left side of the network (Figure 5), a previous study (Mao and Zhong, 2006) showed that once the expression of plasma membrane $\mathrm{H}^{+}$-ATPase is suppressed, cordycepin production decreases, and ATP accumulates. The genes CCM_01006 (CMsI: 136.4,CMsII: 323.5) and CCM_08873 (CMsI: 333.0, CMsII: 975.5), annotated as plasma membrane $\mathrm{H}^{+}$-ATPase, were significantly up-regulated, which matched those of the previous report. CCM_06062, the significantly up-regulated putative DEG in C. militaris, was annotated as

TABLE 2 | Transcription factors of DEGs from FTFD.

\begin{tabular}{|c|c|c|c|}
\hline Transcription factors families & Determined & Undetermined & Total \\
\hline APSES & & 1 & 1 \\
\hline Heteromeric CCAAT factors & 1 & & 1 \\
\hline NDT80/PhoG like DNA-binding & 1 & & 1 \\
\hline Negative transcriptional regulator & 1 & & 1 \\
\hline YL1 nuclear protein & 1 & & 1 \\
\hline AT-rich interaction region & 1 & & 1 \\
\hline Bromodomain transcription factor & 1 & & 1 \\
\hline p53-like transcription factor & 1 & & 1 \\
\hline Centromere protein $\mathrm{B}$, DNA-binding region & 1 & & 1 \\
\hline Zinc finger, DHHC-type & 1 & & 1 \\
\hline Forkhead & & 1 & 1 \\
\hline bHLH & 2 & & 2 \\
\hline GATA type zinc finger & 1 & 1 & 2 \\
\hline Homeobox & 2 & & 2 \\
\hline Bacterial regulatory protein & & 2 & 2 \\
\hline Tubby transcription factors & 1 & 1 & 2 \\
\hline Myb & 2 & & 2 \\
\hline Lambda repressor-like, DNA-binding & 1 & 1 & 2 \\
\hline Zinc finger, CCHC-type & 2 & & 2 \\
\hline Winged helix repressor DNA-binding & 1 & 4 & 5 \\
\hline bZIP & 6 & & 6 \\
\hline Helix-turn-helix & 5 & 1 & 6 \\
\hline $\mathrm{C} 2 \mathrm{H} 2$ zinc finger & 6 & 2 & 8 \\
\hline Nucleic acid-binding, OB-fold & 7 & 1 & 8 \\
\hline Homeodomain-like & 8 & 2 & 10 \\
\hline HMG & 12 & 3 & 15 \\
\hline Zn2Cys6 & 20 & 1 & 21 \\
\hline
\end{tabular}

pyruvate kinase (classified as EC 2.7.1.40) according to sequence homology analysis. Its highly up-regulated transcriptional level will lead to the function of improving ATP generation. Since adenine could be obtained by the over-dephosphorylation of ATP, CCM_06062 indirectly increased the production of adenine, which is a plausible candidate substance of cordycepin. Other than adenine, AMP was also a plausible candidate substrate of cordycepin because previous report showed that adenylate kinase was a key enzyme in cordycepin synthesis (Kuo et al., 2015). Five genes were annotated as adenylate kinase (classified as EC 2.7.4.3) in this study. The CCM_03940 was the only significantly up-regulated one at the transcriptional level. CCM_01353, identified as a unique adenylate kinase gene in C. militaris in a previous report (Vongsangnak et al., 2017), along with the other three genes, both showed no significant difference between the experimental group and control. Another enzyme verified (Kuo et al., 2015) to be involved in cordycepin synthesis was adenine phosphoribosyltransferase (APRTase) (EC 2.4.2.7), which transforms adenine into AMP. The transcriptional level of its annotated gene CCM_00088 was 145.2 in the treatment group and 85.9 in the control, which was only $69 \%$ upregulated in this study.

From the right side of the network (Figure 5), histidine metabolism was predicted to be involved because it is a bypass pathway of adenine formation. The transcriptional level of DEG CCM_06768, which was annotated as denylosuccinate synthase (EC 6.3.4.4) and could transform IMP into adenylosuccinate, was significantly up-regulated (CMsI: 102.4, CMsII: 315.3, 1.6fold). For its downstream reaction, the gene CCM_05789 (the expressed enzyme classified as EC 4.3.2.2), for AMP formation from adenylosuccinate, showed no transcriptional difference between samples. However, its transcriptional level was higher than that of $90 \%$ of the DEGs (CMsI: 130.9, CMsII: 122.1). The gene CCM_02717, annotated as adenosine kinase (EC 2.7.1.20) and takes part in the transformation from AMP to adenosine, was similar to the gene CCM_05789. Its transcriptional level was also relatively high (CMsI: 492.9, CMsII: 446.2, higher than 99.98\% of the DEGs), while no obvious difference was found between samples.

As followed, four genes were annotated as adenosine deaminase (EC 3.5.4.4), while only one DEG, CCM_07799 (CMsI: 113.8, CMsII: 263.8, 1.2-fold), was significantly upregulated in the transcriptome data. A previous study speculated that the synthesis location for cordycepin and purine is separated (Lennon and Suhadolnik, 1976). The up-regulation of CCM_07799 indicated that adenosine deaminase may improve cordycepin production by converting adenosine into inosine in the pathway of cytosol RNA editing.

Finally, the network connected to the cordycepin biosynthesis cluster. The transcriptional levels of CCM_04436, annotated as cns1 (CMsI: 338.3, CMsII: 958.7, 2.8-fold), and CCM_04437, annotated as cns2 (CMsI: 709.0, CMsII: 2194.3, 3.1-fold), were both significantly up-regulated at similar levels. However, the transcriptional level of the gene CCM_04438, annotated as cns3 (CMsI: 143.5, CMsII: 238.4, 1.7-fold), was only slightly increased. $3^{\prime}$-AMP could be also produced by phosphoesterase (EC 3.1.4.16) from $2^{\prime}, 3^{\prime}$-cAMP, which was generated during 


\section{L-alanine}

\section{Energy generation}

\begin{tabular}{|c|}
\hline Pyruvate kinase \\
CCM_06062
\end{tabular}

Plasma membrane $\mathrm{H}^{+}$-ATPase

CCM_01006, CCM_08873

$91.5 \% \quad 97.3 \% \quad 97.8 \% \quad 99.5 \%$

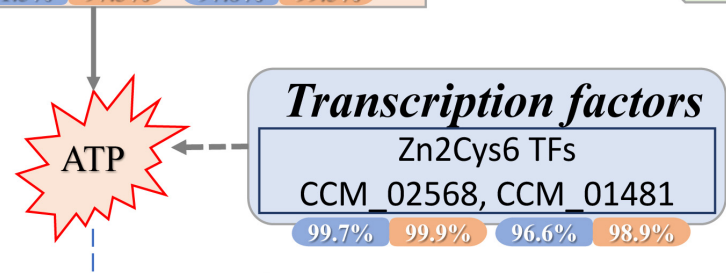

\section{Energy|consumption}

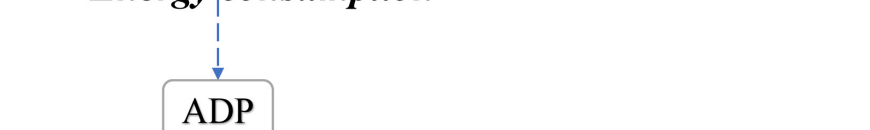

Amino acid interconversion

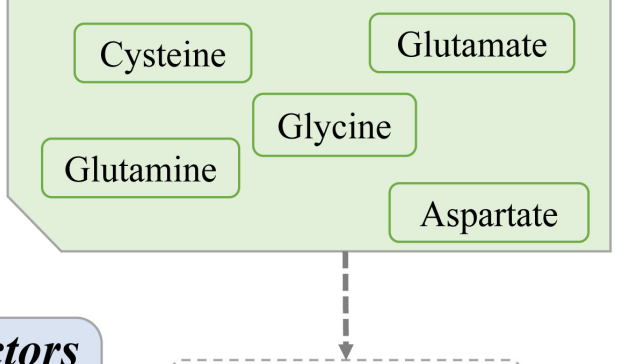

Histidine metabolism

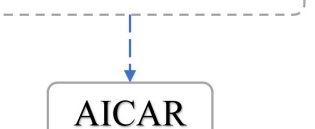

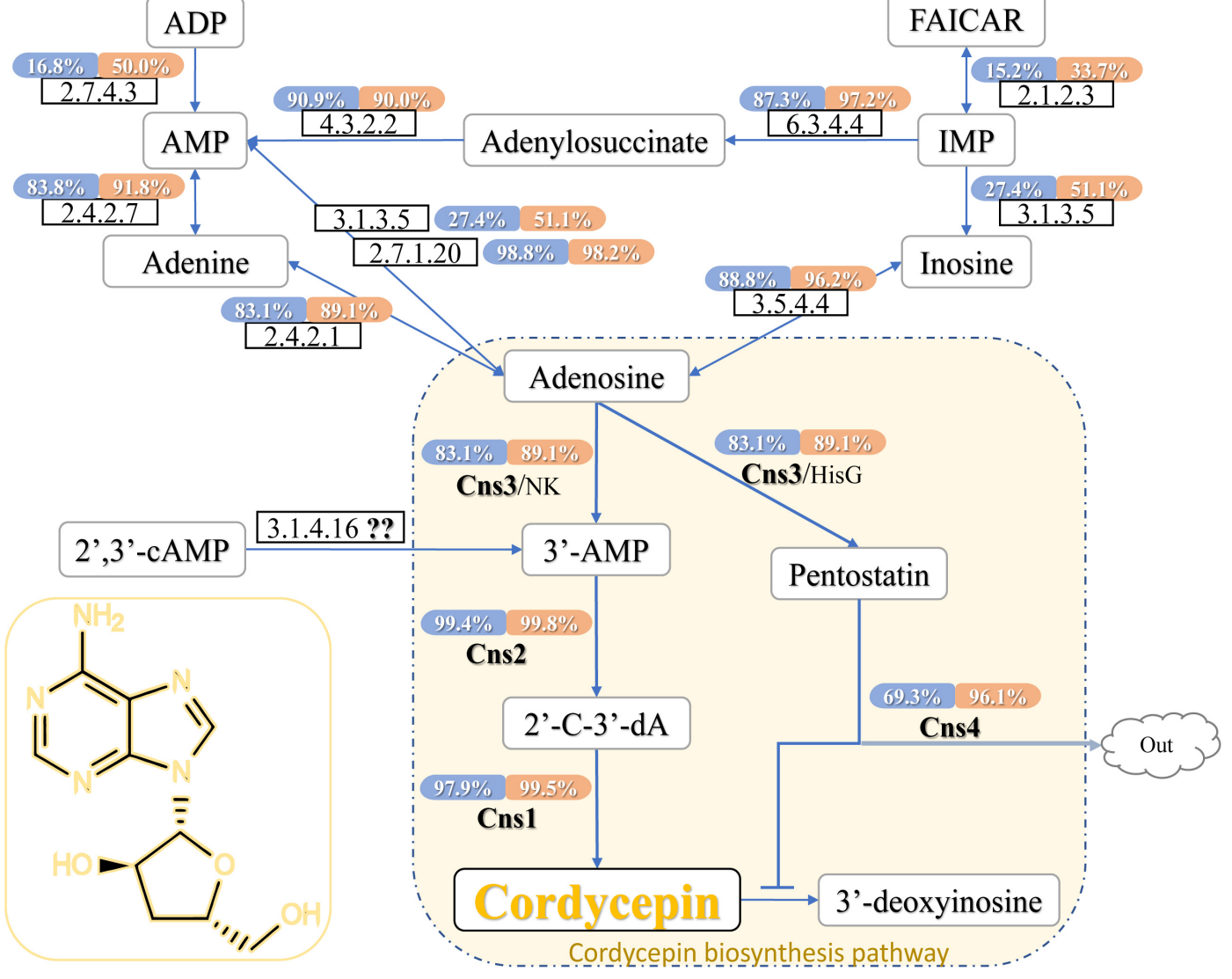

FIGURE 5 | Cordycepin metabolism and biosynthesis pathway in C. militaris. The percentage showed in coloring indicates the ratio that the transcription level of this gene was higher than how many genes of DEGs in samples (CMsl, blue; CMsll, orange). The dotted lines indicate the indirect effects. The solid arrows indicate direct action. TFs, transcription factors; AICAR, 5-aminoimidazole-4-carboxamide ribonucleotide; FAICAR, 5-formamidoimidazole-4-carboxamide ribotide; IMP, inosine monophosphate; ATP, adenosine triphosphate; ADP, adenosine diphosphate; AMP, adenosine monophosphate; 2' $3^{\prime}$-cAMP, $2^{\prime}, 3^{\prime}$-cyclic monophosphate; $3^{\prime}$-AMP, adenosine-3'-phosphate; 2'-C-3'-dA, 2'-carbonyl-3'-deoxyadenosine. 
TABLE 3 | Genes involved in putative cordycepin metabolic network.

\begin{tabular}{|c|c|c|}
\hline Enzymes & EC number & $\begin{array}{l}\text { Gene ID (Transcriptional level } \\
\text { CMsII/CMsl, log2FoldChange } \\
\text { (CMsII/CMsI)) }\end{array}$ \\
\hline $\begin{array}{l}\text { Adenylosuccinate } \\
\text { synthase }\end{array}$ & 6.3 .4 .4 & $\begin{array}{l}\text { CCM_06768(315.3/102.4, 1.62) } \\
\text { CCM_07353(2.7/1.8, 0.60) }\end{array}$ \\
\hline Adenylosuccinate lyase & 4.3.2.2 & CCM_05789(122.1/130.0,-0.10) \\
\hline Pyruvate kinase & 2.7 .1 .40 & CCM_06062(228.9/89.6, 1.35) \\
\hline Adenylate kinase & 2.7 .4 .3 & $\begin{array}{l}\text { CCM_01353(33.1/42.8, -0.37), } \\
\text { CCM_02335(34.2/33.4, 0.04), } \\
\text { CCM_03940(25.4/6.1, 2.07) } \\
\text { CCM_04983(31.7/59.1, 0.81), } \\
\text { CCM_07479(48.2/38.3, 0.33) }\end{array}$ \\
\hline Adenosine kinase & 2.7 .1 .20 & CCM_02717(446.2/493.0, -0.14) \\
\hline Adenosine deaminase & 3.5.4.4 & $\begin{array}{l}\text { CCM_02911(17.5/10.5, 0.74), } \\
\text { CCM_07169(64.6/53.9, 0.43), } \\
\text { CCM_07799(263.8/113.8, 1.21)a, } \\
\text { CCM_09449(60.0/63.1, -0.07) }\end{array}$ \\
\hline $\begin{array}{l}\text { Adenine } \\
\text { phosphoribosyltransferase }\end{array}$ & 2.4.2.7 & $\begin{array}{l}\text { CCM_00088(145.2/85.9, 0.76), } \\
\text { CCM_02051(0.78/0.82, -0.08) }\end{array}$ \\
\hline 5'-Nucleotidase & 3.1.3.5 & $\begin{array}{l}\text { CCM_00622(26.2/11.9, 1.14), } \\
\text { CCM_02830(0.27/0.89, -1.72), } \\
\text { CCM_02831(11.8/10.4, 0.18), } \\
\text { CCM_04038(3.2/0.01, 8.31), } \\
\text { CCM_04644(31.6/23.5, 0.43), } \\
\text { CCM_07972(1.2/11.6, -3.27) }\end{array}$ \\
\hline $\begin{array}{l}\text { Purine-nucleoside } \\
\text { phosphorylase }\end{array}$ & 2.4 .2 .1 & $\begin{array}{l}\text { CCM_04505(25.3/13.1, 0.94), } \\
\text { CCM_04506(113.0/83.3, 0.44) }\end{array}$ \\
\hline IMP cyclohydrolase & 2.1 .2 .3 & CCM_07593(13.8/5.2, 1.40) \\
\hline $\begin{array}{l}\text { Cordycepin synthetase } \\
1\end{array}$ & Cns1 & CCM_04436(958.7/338.3, 1.50) \\
\hline $\begin{array}{l}\text { Cordycepin synthetase } \\
2\end{array}$ & Cns2 & CCM_04437(2194.3/709.0, 1.63) \\
\hline $\begin{array}{l}\text { Cordycepin synthetase } \\
3\end{array}$ & Cns3 & CCM_04438(238.4/143.5, 0.73) \\
\hline $\begin{array}{l}\text { Cordycepin synthetase } \\
4\end{array}$ & Cns4 & CCM_04439(260.6/50.6, 2.36) \\
\hline $\begin{array}{l}\mathrm{H}^{+} \text {-transporting } \\
\text { ATPase }\end{array}$ & 3.6.3.14 & CCM_01006(323.5/136.4, 1.25) \\
\hline $\begin{array}{l}\mathrm{H}^{+} \text {-transporting } \\
\text { ATPase }\end{array}$ & 3.6.3.6 & CCM_08873(975.5/333.0, 1.55) \\
\hline
\end{tabular}

a Up-regulated DEGs.

mRNA degradation. Though the phosphoesterase gene was not determined in C. militaris, $2^{\prime}, 3^{\prime}$-cAMP has been verified to be involved in cordycepin production (Xia et al., 2017).

Besides, 40 of the DEGs (40/983, 4\%) were major facilitator superfamily (MFS) transporters (Supplementary Table S8), and 16 of the DEGs (16/983, 1.6\%) were ATPbinding cassette (ABC) transporters involved in metabolism (Supplementary Table S9). Some of these transporters might regulate other genes or transport other materials to improve the cordycepin production and consequently be involved in amino acid interconversion and cordycepin metabolism in vivo. However, multiple overlapping activities could also promote transporters to interfere in the metabolism of cordycepin and its possible derivatives; further validation of the relation between L-alanine and these transporters remains to be performed in the future.

\section{Validation of RNA-Seq by qRT-PCR}

To further verify the RNA sequencing data, 13 genes represented four different pathways discussed in this study were selected to perform qRT-PCR validation. The qRT-PCR results (Figure 6 and Supplementary Table S1) showed the differential expression pattern of these genes and further indicated the reliability of RNA-Seq data.

Of these genes, seven genes, which were predicted to participate in cordycepin metabolism as described in Figure 5, CCM_04505 (coding gene of EC 2.4.2.1), CCM_03940 (EC 2.7.4.3), CCM_04644 (EC 3.1.3.5), and CCM_05789 (EC 4.3.2.2), took part in purine metabolism and were responsible for the conversion between ADP, AMP, adenosine, and adenylosuccinate (Figure 5). Though the fold changes of these genes in qRT-PCR were slightly different from the RNA-Seq data, they shared a similar up-regulated tendency. Also, CCM_06062 (annotated as pyruvate kinase), regarded to be involved in energy generation, and CCM_01481 and CCM_02568 (both annotated as Zn2Cys6type TFs), regarded to play roles in metabolite regulation, have shown significant consistency $(P<0.01)$ of the fold change between qRT-PCR and RNA-Seq results.

Cordycepin, as the analog of adenosine, is capable of blocking the synthesis of DNA or RNA in inflammatory cells. Nevertheless, a high dose of cordycepin showed no obvious effect on mycelial growth in $C$. militaris. The protein Cns3 was also able to synthesize pentostatin, a purine analog that functions as a safeguard of cordycepin to balance its in vivo concentration to avoid the toxic effect from a high intracellular level of cordycepin (Xia et al., 2017). The transcriptional level of gene cns 3 showed no difference between groups. Four genes [CCM_00136 (CMsI: 19.2, CMsII: 85.8), CCM_05735 (CMsI: 72.0, CMsII: 169.0), CCM_06273 (CMsI: 9.5, CMsII: 49.1), and CCM_07510 (CMsI: 71.5, CMsII: 228.4)], which were involved in the repair mechanism of the synthesis-dependent strand annealing in the homologous recombination pathway (KEGG ko03440), were significantly up-regulated in RNA sequencing data and the qRT-PCR results. This might indicate that the powerful DNA repair mechanism in C. militaris neutralized the toxicity of cordycepin, which causes DNA breaks.

Two other genes, CCM_06968 (Cdc24, CMsI: 53.2, CMsII: 19.0) and CCM_04351 (Flo11, CMsI: 19.4, CMsII: 710.1), which were distinctly different at the transcriptional level and annotated to join in the mitogen-activated protein kinase signaling pathway ko04011, were randomly picked to be verified by qRT-PCR. Their qRT-PCR data were also consistent with the RNA-Seq result.

Overall, these qRT-PCR results showed that the DEGs transcription level was consistent with the data obtained by transcriptome analysis.

\section{Validation of RNA-Seq by Overexpression of TFs in vivo}

According to the RNA-Seq and qRT-PCR in this study, Zn2Cys6 transcript factors were predicted to be one of the key roles to improve cordycepin production. The most relevant genes in this TF family, which might affect amino acid metabolism or cordycepin production, were CCM_02568 and CCM_01481. To 


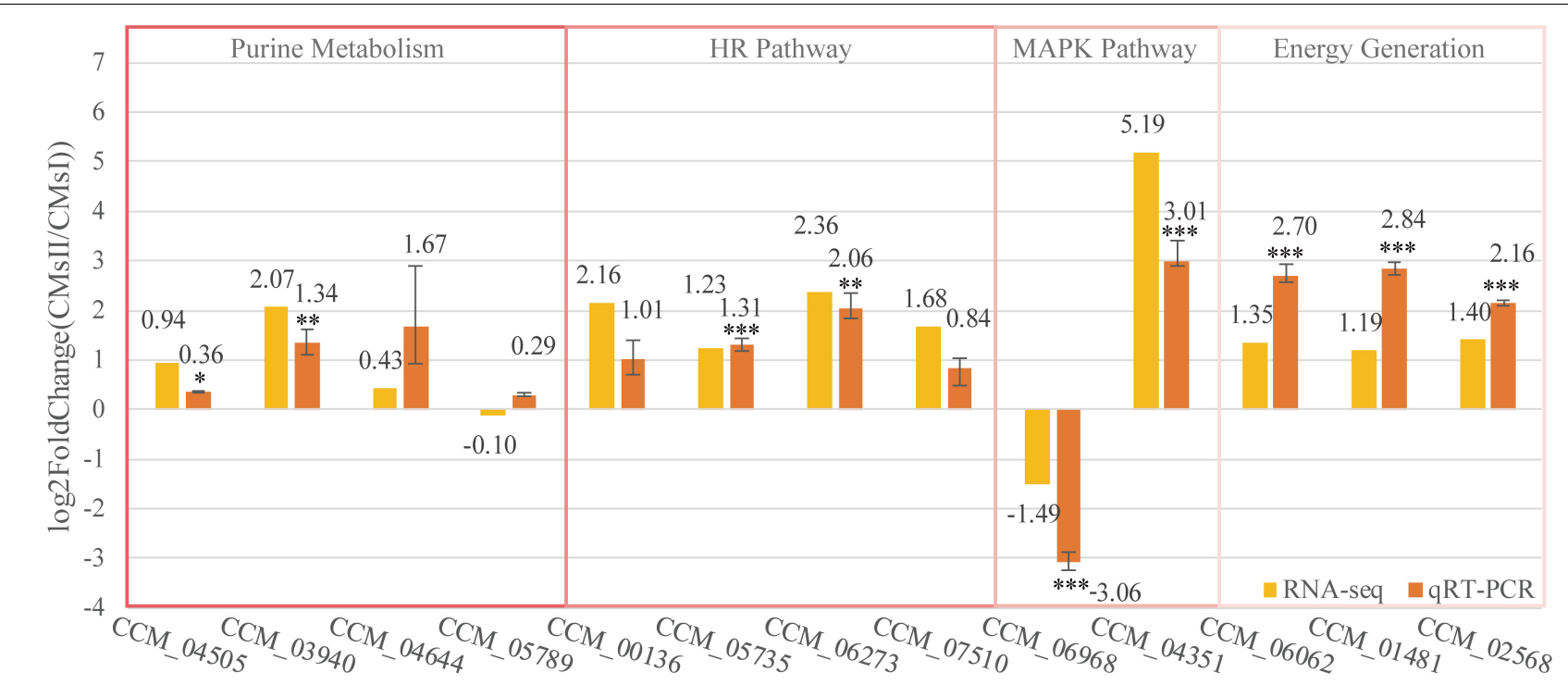

FIGURE 6 | Quantitative real-time PCR validation of DEGs. The qRT-PCR was carried out in biological triplicate. Data were represented as the mean \pm SEM $(n=9)$. Statistical analyses were performed using $t$-tests $\left({ }^{\star} p<0.05,{ }^{\star \star} p<0.01\right.$, and $\left.{ }^{\star \star \star} p<0.001\right)$. The yellow bars (RNA-seq) were represented in the log2 fold change (CMsll/CMsl) of transcriptome data while the orange bars (qRT-PCR) were represented in the log2 regulation of qRT-PCR data.

further verify their function, two vectors with the full-length genes under control by a PtrpC promoter were constructed (Figure 7A) to overexpress CmTf1 and CmTf2 individually. These overexpressed fragments of CmTf1/2 were transferred into C. militaris genome by ATMT to obtain the CmTf1/2 overexpressed strains and renamed as CM10Tf1/CM10Tf2. The growth rate and pigment accumulation (Figure 7B) showed no obvious difference between the wild-type CM10 and CM10Tf1/CM10Tf2.

Shake-flask fermentation was performed to detect the cordycepin concentration. After culturing for 5 days, L-alanine was added into the fermentation. Mediums and mycelium were collected to perform extraction and detection. As Figure 7C showed, the cordycepin yield of CmTf1/2 overexpressed strains were higher than the wild type. The highest yield of cordycepin is $99 \mathrm{mg} / \mathrm{L}$, which is triple than the wild type in the fermentation medium, while the highest yield is $97 \mathrm{ng} / \mathrm{g}$ in the mycelium.

\section{DISCUSSION}

Previous reports (Kang et al., 2014; Zhang et al., 2016) showed that cordycepin production could be increased by adding mineral salts, amino acids, or nucleoside analog. Among these additives, L-alanine was proven to have the best effect on yielding cordycepin (Kang et al., 2014). In this study, to reveal the metabolic pathway of cordycepin, L-alanine was supplemented as an inducer to improve the production of cordycepin in C. militaris. After culturing in the liquid static medium for 30 days, the yield of cordycepin was 2.1 times higher than the one without L-alanine supplementary (Figure 1B). Besides, the stage of color change and primordium formation was brought forward by the supplementary. Since cordycepin is a colorless nucleoside analog, the color distinctions in growth characteristics might be due to a higher concentration of pigment accumulation (Figure 1A). Generally, the primordium is formed by gathering a certain amount of mycelium in C. militaris. The improved growth rate of mycelia will accumulate more hyphae so that the primordium will generate beforehand. The earlier primordium formation of $C$. militaris indicated that $\mathrm{L}$-alanine might increase cordycepin production by improving the mycelia growth rate.

To figure out the metabolic pathway from the additive L-alanine to cordycepin, four samples were collected and transcriptome sequencing was performed. The transcription data were further elaborated with annotations from the reference database of C. militaris and showed that approximately $80 \%$ of the genes (Supplementary Table S3) were expressed during the mycelial stage of $C$. militaris. To further verify the data, the read distributions, the Pearson correlation coefficient between expression values across samples, and hierarchical clustering between four samples were calculated (Figures 2A-C). They show which samples shared the same culture condition, which could be considered as reliable biological replicates. The DEG analysis results shown in the volcano plot and heatmap (Figures 2E,F) revealed that adding L-alanine may only induce a few genes to regulate the metabolism in vivo, which reveals a possibility to narrow down the DEG targets to figure out which specific genes were taking part in the synthesis and metabolism of cordycepin. To further analyze and to classify the DEGs, the sequencing data were uploaded to the GO and KEGG database (Figure 3). GO classification results of DEGs indicated that the additive might improve the metabolic process to produce more metabolites and consequently activate membrane transportation to avoid unaffordable cordycepin overproduction.

Meanwhile, KEGG classification showed that most DEGs were relevant to the pathway of individual growth and metabolism, 
A

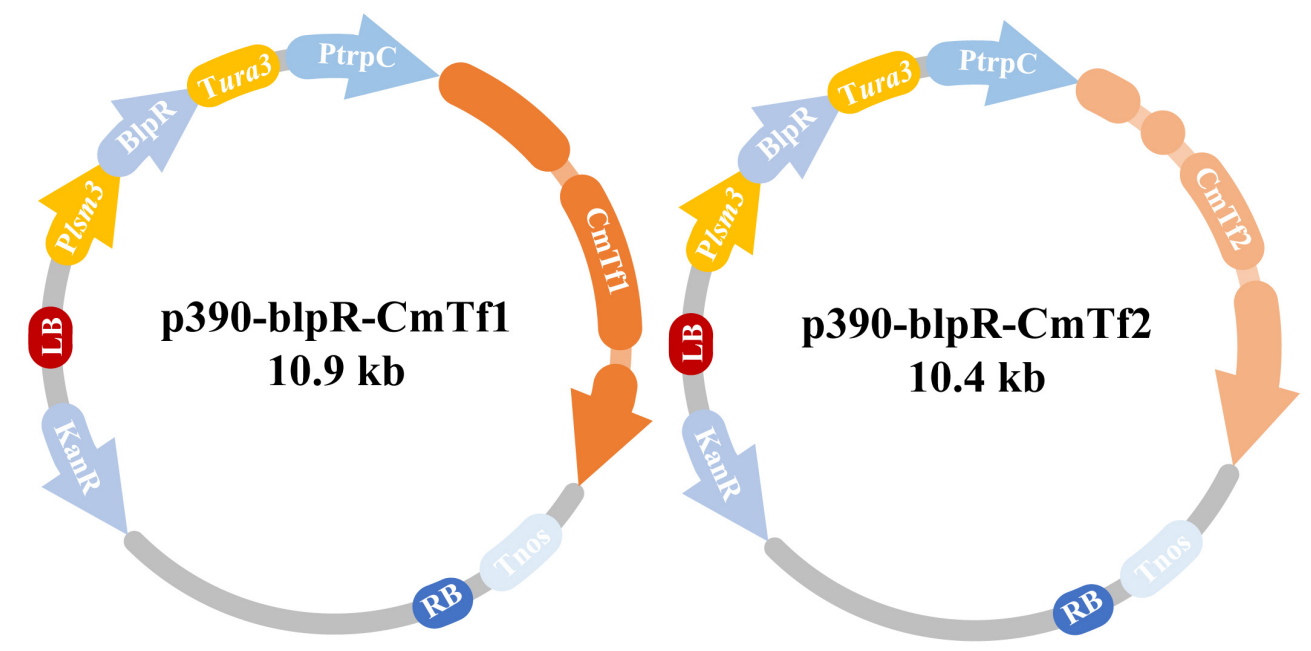

B
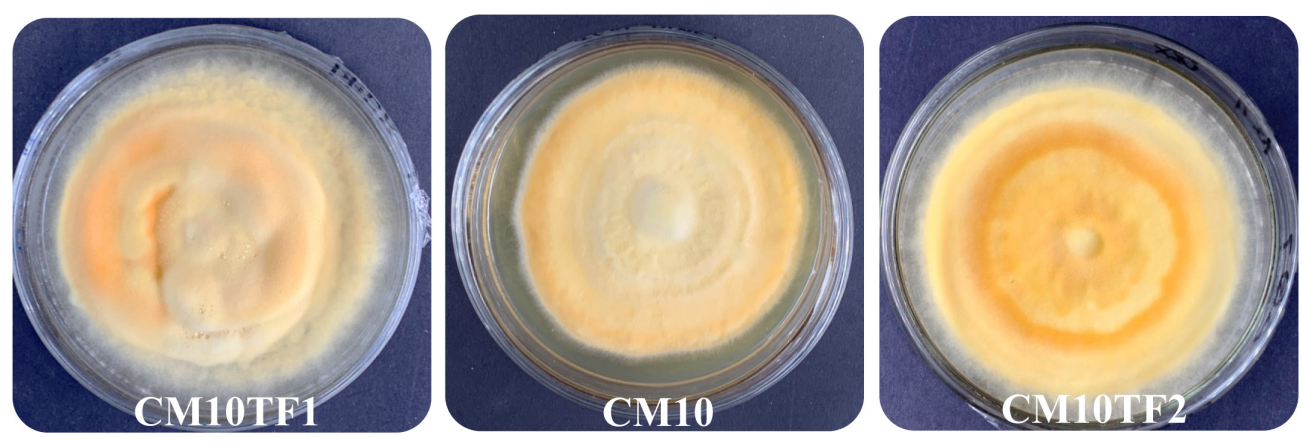

C

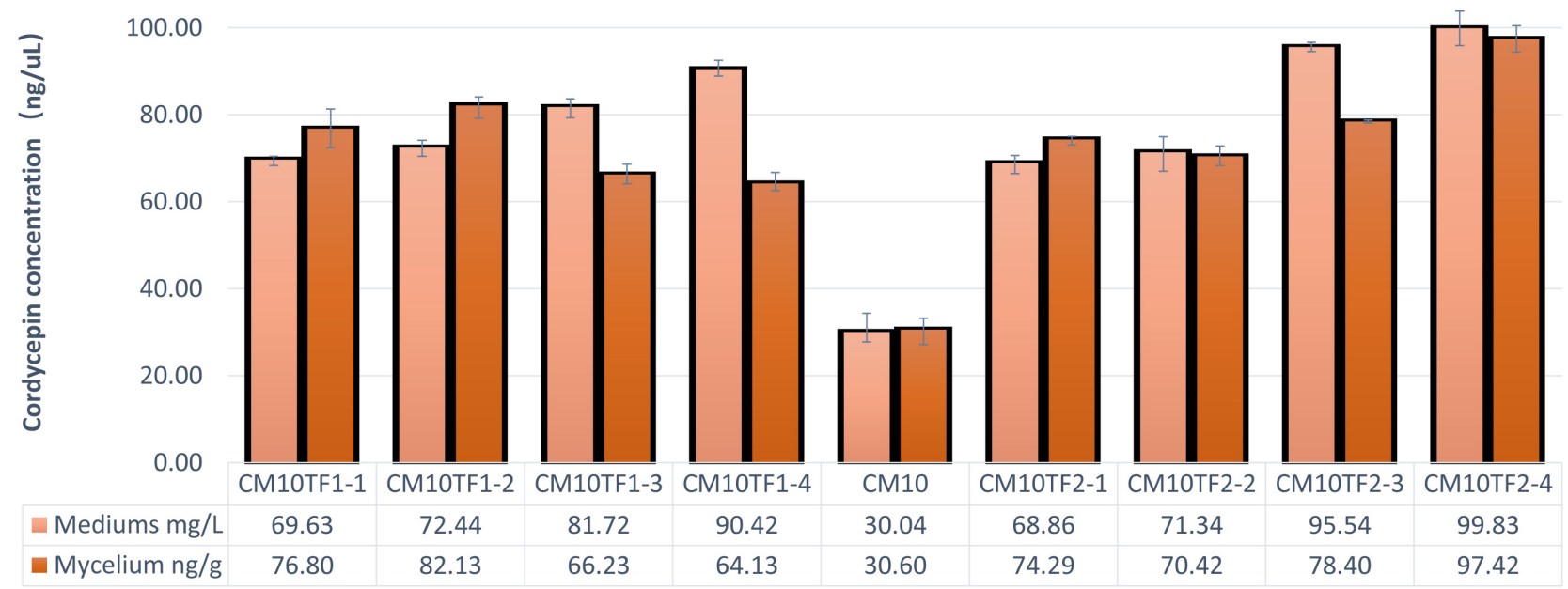

FIGURE 7 | Overexpression of CmTf1 and CmTf2 in C. militaris. (A) Schematic diagram of CmTf1/2 overexpression vector p390-blpR-CmTf1/2. (B) Growth characteristics of $\mathrm{C}$. militaris CM10TF1 (left), CM10 (middle), and CM10TF2 (right) on PDA plate. (C) Cordycepin production in mediums and mycelium of the C. militaris $\mathrm{CM} 10$ and $\mathrm{CM} 10 \mathrm{TF} 1 / 2$ after 8 days of flask-shake culturing.

which indicated that supplementation with L-alanine might affect the specific amino acid metabolism and energy metabolism. Since amino acids were reported to be involved in the purine pathway
(Zheng et al., 2011), to figure out the relationship between L-alanine addition and cordycepin production, metabolic substance transformation was rearranged and showed in a 
network map. As shown in Figure 4, L-cysteine and L-glutamate were shown as the most important substances while foreign L-alanine was absorbed and included in the metabolism. This indicated that additives like L-alanine may improve cordycepin production by imparting amino acid conversion. Besides, significant changes in the KEGG pathway ko01100 were confirmed as previously mentioned (Vongsangnak et al., 2017). Glycolysis, the pentose phosphate pathway, and the citrate cycle were involved in the biosynthesis of cordycepin by supplying energy for any relevant bioactivities. This indicated that the addition of L-alanine increased energy molecule production and further improved the cordycepin production.

Since few transcription factors were reported to be involved in cordycepin production, the DEGs were uploaded into FIFD and further verified by InterPro to find relevant TF families and genes. The largest TF family was Zn2Cys6, which was also reported as an important part of the fruit body formation of C. militaris (Zheng et al., 2011). The most relevant genes in this TF family, which might affect amino acid metabolism or cordycepin production, were CCM_02568 and CCM_01481. Since these genes are highly up-regulated transcripts compared to the treatment, an overexpression was performed in $C$. militaris CM10. The overexpression highly improved the cordycepin production with the addition of L-alanine (Figure $7 \mathrm{C}$ ) but did not interfere with the mycelia growth rate and pigment accumulation (Figure 7B). This indicated that these transcription factors may not be involved in major physiological activity but only join in specific secondary metabolites to improve cordycepin production. The gene silencing of TFs could further study their function, but the efficient gene inhibition system has not been established in C. militaris. Disruption (Chen et al., 2018; Lou et al., 2018) of the genes is another option. The deletion of the transcription factor will lead to multiple effects, because TFs may bind to multiple DNA elements of different genes in different space-time stages. It is hard to build a direct relationship between absent TFs and cordycepin production. Nevertheless, detailed biological functions of CmTf1 and CmTf2 need further researches, such as multiple gene editing or chromatin immunoprecipitation technology. Though evidence was not enough to fulfill the detail of how they regulate the metabolism in this study, they lead to new genome-editing targets for cordycepin overproduction. Moreover, combined with the GO and KEGG results, some genes of the 40 MFS (Supplementary Table S8) and 16 ABC transporters (Supplementary Table S9) might play important roles in amino acid interconversion and cordycepin metabolism in vivo.

Even though cordycepin is a purine nucleoside analog, the prediction of the cordycepin pathway in purine metabolism lacked detail. Based on the KEGG ko00230 and cordycepin synthetic pathway in the previous report, results in this study were comprehensively analyzed and the draft of the whole cordycepin metabolic network in C. militaris was drawn (Figure 5).

On the one hand, as the KEGG annotation result and the network map shown in Figure 4, L-alanine could improve the cordycepin production by activating the amino acid interconversion. Five amino acids (cysteine, glutamate, glutamine, glycine, and aspartate) showed a clear function in the interconversion and directly stimulated the metabolic substance conversion of histidine metabolism so that it indirectly took part in the cordycepin synthetic pathway.

As a bypass pathway of adenine formation, histidine metabolism was involved in the cordycepin pathway by offering the precursor IMP. Adenylosuccinate synthase (EC 6.3.4.4) gene CCM_06768 could transform IMP into adenylosuccinate, and then the adenylosuccinate lyase (EC 4.3.2.2) gene CCM_05789 will perform the reaction of producing AMP. CCM_06768 was significantly up-regulated while CCM_05789 was not, but the transcriptional level of CCM_05789 was higher than that of 90\% DEGs. The gene CCM_00088, annotated as APRTase (EC 2.4.2.7) and conducted the reaction from adenine into AMP, also had a high transcriptional level but only showed slightly up-regulated while compared to the control. The high transcriptional level of these genes indicated that they might be involved in cordycepin synthesis, even though they were not significantly up-regulated in the experimental group with L-alanine addition. Previous studies have noted the importance of adenylate kinase (Kuo et al., 2015) and APRTase (Xiong et al., 2010) in cordycepin synthesis. In this study, adenylate kinase (EC 2.7.4.3) gene CCM_03940 was significantly up-regulated, which might accelerate the conversion from ADP to AMP. This result indicated that the adenylate kinase was one of the rate-limiting enzymes in the cordycepin production pathway.

On the other hand, not only the phenotypic character but also the KEGG classification and ko01100 results indicated that the energy generation was acting an important role in the cordycepin production. From a cellular perspective, pyruvate not only joins in ketone body formation as a precursor but also takes part in ATP generation. In this study, high-level gene expression of pyruvate kinase gene CCM_06062 indicated that it joined in cordycepin metabolism by improving ATP generation. As consistent with the previous report (Mao and Zhong, 2006), $\mathrm{H}^{+}$-ATPase genes CCM_01006 and CCM_08873 were highly upregulated to improve the cordycepin production. This discovery accords with the GO classification and TFs results, suggesting that L-alanine activates additional expression of transporters known to affect energy generation and metabolic substance conversion.

As mentioned above (Lennon and Suhadolnik, 1976), the direct substrate of cordycepin was adenosine. In the purine metabolism, adenosine could be synthesized from three reactions. The first one is conducted by adenosine kinase (EC 2.7.1.20). Although the gene expression level of CCM_02717 did not show significant difference among samples, its transcriptional level was higher than 99.98\% DEGs, which indicated that it might be the key enzyme for cell growth rather than the key enzyme in improving cordycepin production by L-alanine. The second pathway was synthesis of adenosine from adenine by purine-nucleoside phosphorylase. However, the transcriptional level of involved gene CCM_04506 (EC 2.4.2.1) was similar to the gene CCM_05789 (EC 4.3.2.2), showing no significant difference between samples. The last one performed a conversion between inosine and adenosine. The enzyme adenosine deaminase was reported to convert adenosine into inosine in the pathway of cytosol RNA editing. Yet, the high transcript gene CCM_07799 
(EC 3.5.4.4) might catalyze the reverse reaction to improve the cordycepin production in this study.

Cordycepin was reported to be synthesized from adenosine by a three-gene cassette (Xia et al., 2017). Adenosine is converted to $3^{\prime}$-AMP by Cns3, which also possesses the function of converting adenosine to the cordycepin protector pentostatin. Cordycepin is synthesized after $3^{\prime}$-AMP is converted to the intermediate $2^{\prime}$-carbonyl- $3^{\prime}$-deoxyadenosine $\left(2^{\prime}-\mathrm{C}-3^{\prime}-\mathrm{dA}\right)$ by the protein complex Cns1-Cns2. In this study, gene cns1 and cns2 were significantly up-regulated. This result suggested that the dosage of additive L-alanine in this study was enough to sustain improvement of the cordycepin production. Contrary to expectations, this study showed that gene cns3 was not significantly up-regulated between samples, indicating that adding L-alanine could not significantly activate the expression of Cns3. One possible hypothesis is that most of substrate $3^{\prime}$-AMP is synthesized from $2^{\prime}, 3^{\prime}$-cAMP through the undetermined enzyme EC 3.1.4.16 during mRNA degradation.

Above all, the combination of transcriptome data in this study and the data from previous reports revealed a reliable metabolic network map of cordycepin production affected by L-alanine addition in the fermentation of $C$. militaris. In brief, the addition of L-alanine increased the cordycepin yield by activating the pathway of energy generation and amino acid interconversion. In the biopathway of energy generation, the activated pyruvate kinase and plasma membrane $\mathrm{H}^{+}$-ATPase directly increased the accumulation of ATP, while the Zn2Cys6 TFs (CCM_02568 and CCM_01481) promoted activities in some unknown ways to generate more energy. The consumption of extra ATP could generate a high amount of ADP and AMP, which are precursors of adenosine. In the other biopathway, L-alanine joined in the interconversion of amino acids, the high amount of L-alanine accelerates the formation of amino acids. Through the histidine metabolism, these amino acids indirectly promoted the accumulation of IMP, which is also the precursor of adenosine. The enzymes of the reactions between AMP, IMP, and their intermediate products (adenine, inosine, and adenylosuccinate) to adenosine performed an active stage because of the sufficient AMP and IMP. Thus, the production of cordycepin was increased by excess adenosine and energy.

\section{CONCLUSION}

We presented the transcriptome analysis of the metabolic network of cordycepin in C. militaris activated by L-alanine addition. Up-regulating the transcriptome level of genes involved

\section{REFERENCES}

Ashburner, M., Ball, C. A., Blake, J. A., Botstein, D., Butler, H., Cherry, J. M., et al. (2000). Gene Ontology: tool for the unification of biology. Nat. Genet. 25, 25-29. doi: $10.1038 / 75556$

Audic, S., and Claverie, J. M. (1997). The significance of digital gene expression profiles. Genome Res. 7, 986-995. doi: 10.1101/gr.7.10.986

Chen, B.-X., Wei, T., Ye, Z.-W., Yun, F., Kang, L.-Z., Tang, H.-B., et al. (2018). Efficient CRISPR-Cas9 gene disruption system in edible-medicinal mushroom in the bio-pathways of energy generation and amino acid conversion was the major reason for the overproduction of cordycepin. Two Zn2Cys6-type transcription factors were verified to be related to the rate-limiting steps by increasing the copy number of their coding genes in C. militaris wild type. As the whole cordycepin metabolic network map was drawn, this is one step toward discovering the flexibility of cordycepin network and providing a reference for genomic editing to improve cordycepin production as well as other compounds of medicinal value in C. militaris.

\section{DATA AVAILABILITY STATEMENT}

The datasets generated for this study can be found in the NCBI Accession: PRJNA413637.

\section{AUTHOR CONTRIBUTIONS}

B-XC performed data analysis and was a major contributor in drafting the work. TW helped revise the manuscript critically for important intellectual content. B-XC and L-NX performed RNA extraction and qRT-PCR. Q-WZ and Z-WY supervised and B-XC in sequencing data analysis. $\mathrm{YZ}$ and $\mathrm{YY}$ performed cordycepin detection. FY performed strain culturing. L-QG and J-FL agreed to be accountable for all aspects of the work in ensuring that questions related to the accuracy or integrity of any part of the work are appropriately investigated and resolved. All authors approved the last version of the manuscript to be published.

\section{FUNDING}

This study was supported by the Key-Area Research and Development Program of Guangdong Province (Grant Nos. 2018B020205001 and 2018B020205003) and the National Natural Science Foundation of China (Grant Nos. 31572178 and 31772373), and the Graduate Students Overseas Joint Education Program of South China Agricultural University (Grant No. 2018LHPY016).

\section{SUPPLEMENTARY MATERIAL}

The Supplementary Material for this article can be found online at: https://www.frontiersin.org/articles/10.3389/fmicb. 2020.00577/full\#supplementary-material

Cordyceps militaris. Front. Microbiol. 9:1157. doi: 10.3389/fmicb.2018. 01157

Cock, P. J. A., Fields, C. J., Goto, N., Heuer, M. L., and Rice, P. M. (2010). The Sanger FASTQ file format for sequences with quality scores, and the Solexa/Illumina FASTQ variants. Nucleic Acids Res. 38, 1767-1771. doi: 10. 1093/NAR/GKP1137

Cohen, N., Cohen, J., Asatiani, M. D., Varshney, V. K., Yu, H.-T., Yang, Y.C., et al. (2014). Chemical composition and nutritional and medicinal value of fruit bodies and submerged cultured mycelia of culinary-medicinal higher 
Basidiomycetes mushrooms. Int. J. Med. Mushrooms 16, 273-291. doi: 10.1615/ IntJMedMushr.v16.i3.80

Cunningham, K. G., Manson, W., Spring, F. S., and Hutchinson, S. A. (1950). Cordycepin, a metabolic product isolated from cultures of Cordyceps militaris (Linn.) link. Nature 166, 949-949. doi: 10.1038/166949a0

Dementhon, K., Saupe, S. J., and Clavé, C. (2004). Characterization of IDI-4, a bZIP transcription factor inducing autophagy and cell death in the fungus Podospora anserina. Mol. Microbiol. 53, 1625-1640. doi: 10.1111/j.1365-2958.2004. 04235.x

Fan, D., Wang, W., and Zhong, J.-J. (2012). Enhancement of cordycepin production in submerged cultures of Cordyceps militaris by addition of ferrous sulfate. Biochem. Eng. J. 60, 30-35. doi: 10.1016/j.bej.2011.09.014

Gajiwala, K. S., and Burley, S. K. (2000). Winged helix proteins. Curr. Opin. Struct. Biol. 10, 110-116. doi: 10.1016/S0959-440X(99)00057-3

Gallegos, M. T., Schleif, R., Bairoch, A., Hofmann, K., and Ramos, J. L. (1997). Arac/XylS family of transcriptional regulators. Microbiol. Mol. Biol. Rev. 61, 393-410.

Gu, Y.-X., Wang, Z.-S., Li, S.-X., and Yuan, Q.-S. (2007). Effect of multiple factors on accumulation of nucleosides and bases in Cordyceps militaris. Food Chem. 102, 1304-1309. doi: 10.1016/j.foodchem.2006.07.018

Hall, D. B., Wade, J. T., and Struhl, K. (2006). An HMG protein, Hmo1, associates with promoters of many ribosomal protein genes and throughout the rRNA gene locus in Saccharomyces cerevisiae. Mol. Cell. Biol. 26, 3672-3679. doi: 10.1128/MCB.26.9.3672-3679.2006

Jin, M. L., Park, S. Y., Kim, Y. H., Oh, J. II, Lee, S. J., and Park, G. (2014). The neuroprotective effects of cordycepin inhibit glutamate-induced oxidative and ER stress-associated apoptosis in hippocampal HT22 cells. Neurotoxicology 41, 102-111. doi: 10.1016/j.neuro.2014.01.005

Kanehisa, M., Araki, M., Goto, S., Hattori, M., Hirakawa, M., Itoh, M., et al. (2007). KEGG for linking genomes to life and the environment. Nucleic Acids Res. 36, D480-D484. doi: 10.1093/nar/gkm882

Kang, C., Wen, T.-C., Kang, J.-C., Meng, Z.-B., Li, G.-R., and Hyde, K. D. (2014). Optimization of Large-Scale culture conditions for the production of cordycepin with Cordyceps militaris by liquid static culture. Sci. World J. 2014, 1-15. doi: 10.1155/2014/510627

Kim, D., Langmead, B., and Salzberg, S. L. (2015). HISAT: a fast spliced aligner with low memory requirements. Nat. Methods 12, 357-360. doi: 10.1038/nmeth. 3317

Kim, H. G., Shrestha, B., Lim, S. Y., Yoon, D. H., Chang, W. C., Shin, D.-J., et al. (2006). Cordycepin inhibits lipopolysaccharide-induced inflammation by the suppression of NF- $\mathrm{B}$ through Akt and p38 inhibition in RAW 264.7 macrophage cells. Eur. J. Pharmacol. 545, 192-199. doi: 10.1016/j.ejphar.2006. 06.047

Kim, S., Park, S.-Y., Kim, K. S., Rho, H.-S., Chi, M.-H., Choi, J., et al. (2009). Homeobox transcription factors are required for conidiation and appressorium development in the rice blast fungus Magnaporthe oryzae. PLoS Genet. 5:e1000757. doi: 10.1371/journal.pgen.1000757

Kong, L., Zhang, Y., Ye, Z.-Q., Liu, X.-Q., Zhao, S.-Q., Wei, L., et al. (2007). CPC: assess the protein-coding potential of transcripts using sequence features and support vector machine. Nucleic Acids Res. 35, W345-W349. doi: 10.1093/nar/ gkm391

Kuo, H.-C., Huang, I.-C., and Chen, T.-Y. (2015). Cordyceps s.l. (Ascomycetes) species used as medicinal mushrooms are closely related with higher ability to produce cordycepin. Int. J. Med. Mushrooms 17, 1077-1085. doi: 10.1615/ IntJMedMushrooms.v17.i11.80

Langmead, B., and Salzberg, S. L. (2012). Fast gapped-read alignment with Bowtie 2. Nat. Methods 9, 357-359. doi: 10.1038/nmeth.1923

Le, J., Liu, J., Bo, Z., Feng, X., Kexue, Y., and Fujiang, W. (2006). The effect of Zn on the $\mathrm{Zn}$ accumulation and biosynthesis of amino acids in mycelia of Cordyceps sinensis. Biol. Trace Elem. Res. 113, 45-52. doi: 10.1385/BTER

Lee, Y.-R., Noh, E.-M., Jeong, E.-Y., Yun, S.-K., Jeong, Y.-J., Kim, J.-H., et al. (2009). Cordycepin inhibits UVB-induced matrix metalloproteinase expression by suppressing the $\mathrm{NF}-\kappa \mathrm{B}$ pathway in human dermal fibroblasts. Exp. Mol. Med. 41, 548-554. doi: 10.3858/emm.2009. 41.8.060

Lennon, M. B., and Suhadolnik, R. J. (1976). Biosynthesis of 3'-deoxyadenosine by Cordyceps militaris. Biochim. Biophys. Acta 425, 532-536. doi: 10.1016/00052787(76)90017-4
Li, B., and Dewey, C. N. (2011). RSEM: accurate transcript quantification from RNA-Seq data with or without a reference genome. BMC Bioinformatics 12:323. doi: 10.1186/1471-2105-12-323

Lian, T., Yang, T., Liu, G., Sun, J., and Dong, C. (2014). Reliable reference gene selection for Cordyceps militaris gene expression studies under different developmental stages and media. FEMS Microbiol. Lett. 356, 97-104. doi: 10. 1111/1574-6968.12492

Livak, K. J., and Schmittgen, T. D. (2001). Analysis of relative gene expression data using real-time quantitative PCR and the 2- $\Delta \Delta$ CT method. Methods 25, 402-408. doi: 10.1006/meth.2001.1262

Lou, H., Ye, Z., Yun, F., Lin, J., Guo, L., Chen, B., et al. (2018). Targeted gene deletion in Cordyceps militaris using the split-marker approach. Mol. Biotechnol. 60, 380-385. doi: 10.1007/s12033-018-0080-9

MacPherson, S., Larochelle, M., and Turcotte, B. (2006). A fungal family of transcriptional regulators: the zinc cluster proteins. Microbiol. Mol. Biol. Rev. 70, 583-604. doi: 10.1128/MMBR.00015-06

Maere, S., Heymans, K., and Kuiper, M. (2005). BiNGO: a Cytoscape plugin to assess overrepresentation of Gene Ontology categories in Biological Networks. Bioinformatics 21, 3448-3449. doi: 10.1093/bioinformatics/bti551

Mao, X.-B., Eksriwong, T., Chauvatcharin, S., and Zhong, J.-J. (2005). Optimization of carbon source and carbon/nitrogen ratio for cordycepin production by submerged cultivation of medicinal mushroom Cordyceps militaris. Process Biochem. 40, 1667-1672. doi: 10.1016/j.procbio.2004.06.046

Mao, X.-B., and Zhong, J.-J. (2006). Significant effect of NH4+ on cordycepin production by submerged cultivation of medicinal mushroom Cordyceps militaris. Enzyme Microb. Technol. 38, 343-350. doi: 10.1016/j.enzmictec.2004. 10.010

Masuda, M., Urabe, E., Honda, H., Sakurai, A., and Sakakibara, M. (2007). Enhanced production of cordycepin by surface culture using the medicinal mushroom Cordyceps militaris. Enzyme Microb. Technol. 40, 1199-1205. doi: 10.1016/J.ENZMICTEC.2006.09.008

Noh, E.-M., Kim, J.-S., Hur, H., Park, B.-H., Song, E.-K., Han, M.-K., et al. (2008). Cordycepin inhibits IL-1 -induced MMP-1 and MMP-3 expression in rheumatoid arthritis synovial fibroblasts. Rheumatology 48, 45-48. doi: 10.1093/ rheumatology/ken417

Park, J., Park, J., Jang, S., Kim, S., Kong, S., Choi, J., et al. (2008). FTFD: an informatics pipeline supporting phylogenomic analysis of fungal transcription factors. Bioinformatics 24, 1024-1025. doi: 10.1093/bioinformatics/btn058

Paterson, R. R. M. (2008). Cordyceps - A traditional Chinese medicine and another fungal therapeutic biofactory? Phytochemistry 69, 1469-1495. doi: 10.1016/j. phytochem.2008.01.027

Pertea, M., Pertea, G. M., Antonescu, C. M., Chang, T.-C., Mendell, J. T., and Salzberg, S. L. (2015). StringTie enables improved reconstruction of a transcriptome from RNA-seq reads. Nat. Biotechnol. 33, 290-295. doi: 10.1038/ nbt. 3122

Raethong, N., Laoteng, K., and Vongsangnak, W. (2018). Uncovering global metabolic response to cordycepin production in Cordyceps militaris through transcriptome and genome-scale network-driven analysis. Sci. Rep. 8, 1-13. doi: 10.1038/s41598-018-27534-7

Robinson, M. D., McCarthy, D. J., and Smyth, G. K. (2010). edgeR: a Bioconductor package for differential expression analysis of digital gene expression data. Bioinformatics 26, 139-140. doi: 10.1093/bioinformatics/btp616

Shannon, P., Markiel, A., Ozier, O., Baliga, N. S., Wang, J. T., Ramage, D., et al. (2003). Cytoscape: a software environment for integrated models of biomolecular interaction networks. Genome Res. 13, 2498-2504. doi: 10.1101/ gr.1239303

Tarazona, S., García-Alcalde, F., Dopazo, J., Ferrer, A., and Conesa, A. (2011). Differential expression in RNA-seq: a matter of depth. Genome Res. 21, 22132223. doi: 10.1101/gr.124321.111

Taylor, M. V., Beatty, K. E., Hunter, H. K., and Baylies, M. K. (1995). Drosophila MEF2 is regulated by twist and is expressed in both the primordia and differentiated cells of the embryonic somatic, visceral and heart musculature. Mech. Dev. 50, 29-41. doi: 10.1016/0925-4773(94)00323-F

Tian, X., Li, Y., Shen, Y., Li, Q., Wang, Q., and Feng, L. (2015). Apoptosis and inhibition of proliferation of cancer cells induced by cordycepin. Oncol. Lett. 10, 595-599. doi: 10.3892/ol.2015.3273

Trapnell, C., Roberts, A., Goff, L., Pertea, G., Kim, D., Kelley, D. R., et al. (2012). Differential gene and transcript expression analysis of RNA-seq experiments 
with TopHat and Cufflinks. Nat. Protoc. 7, 562-578. doi: 10.1038/nprot.2012. 016

Tuli, H. S., Sandhu, S. S., and Sharma, A. K. (2014). Pharmacological and therapeutic potential of Cordyceps with special reference to Cordycepin. 3 Biotech 4, 1-12. doi: 10.1007/s13205-013-0121-9

Vongsangnak, W., Raethong, N., Mujchariyakul, W., Nguyen, N. N., Leong, H. W., Laoteng, K., et al. (2017). Genome-scale metabolic network of Cordyceps militaris useful for comparative analysis of entomopathogenic fungi. Gene 626, 132-139. doi: 10.1016/j.gene.2017.05.027

Xia, Y., Luo, F., Shang, Y., Chen, P., Lu, Y., and Wang, C. (2017). Fungal cordycepin biosynthesis is coupled with the production of the safeguard molecule pentostatin. Cell Chem. Biol. 24, 1-11. doi: 10.1016/j.chembiol.2017. 09.001

Xiong, C., Xia, Y., Zheng, P., Shi, S., and Wang, C. (2010). TMYC developmental stage-specific gene expression profiling for a medicinal fungus Cordyceps militaris. Mycology 1, 25-66. doi: 10.1080/21501201003674581

Yin, Y., Yu, G., Chen, Y., Jiang, S., Wang, M., Jin, Y., et al. (2012). Genomewide transcriptome and proteome analysis on different developmental stages of Cordyceps militaris. PLoS One 7:e51853. doi: 10.1371/journal.pone.005 1853

Zhang, G., Yin, Q., Han, T., Zhao, Y., Su, J., Li, M., et al. (2015). Purification and antioxidant effect of novel fungal polysaccharides from the stroma of Cordyceps kyushuensis. Ind. Crops Prod. 69, 485-491. doi: 10.1016/j.indcrop.2015.03.006

Zhang, Q., Liu, Y., Di, Z., Han, C., and Liu, Z. (2016). The strategies for increasing cordycepin production of Cordyceps militaris by liquid fermentation. Fungal Genomics Biol. 6, 1-5. doi: 10.4172/2165-8056.1000134
Zheng, P., Xia, Y., Xiao, G., Xiong, C., Hu, X., Zhang, S., et al. (2011). Genome sequence of the insect pathogenic fungus Cordyceps militaris, a valued traditional chinese medicine. Genome Biol. 12:R116. doi: 10.1186/gb-2011-1211-r116

Zhou, X., Cai, G., He, Y., and Tong, G. (2016). Separation of cordycepin from Cordyceps militaris fermentation supernatant using preparative HPLC and evaluation of its antibacterial activity as an NAD+-dependent DNA ligase inhibitor. Exp. Ther. Med. 12, 1812-1816. doi: 10.3892/etm.2016.3536

Zhou, X., Gong, Z., Su, Y., Lin, J., and Tang, K. (2009). Cordyceps fungi: natural products, pharmacological functions and developmental products. J. Pharm. Pharmacol. 61, 279-291. doi: 10.1211/jpp.61.03.0002

Conflict of Interest: FY was employed by Guangzhou Alchemy Biotechnology Co., Ltd.

The remaining authors declare that the research was conducted in the absence of any commercial or financial relationships that could be construed as a potential conflict of interest.

Copyright (c) 2020 Chen, Wei, Xue, Zheng, Ye, Zou, Yang, Yun, Guo and Lin. This is an open-access article distributed under the terms of the Creative Commons Attribution License (CC BY). The use, distribution or reproduction in other forums is permitted, provided the original author(s) and the copyright owner(s) are credited and that the original publication in this journal is cited, in accordance with accepted academic practice. No use, distribution or reproduction is permitted which does not comply with these terms. 